\title{
The Proportional Random Allocation Of Indivisible Units
}

\author{
Hervé Moulin \\ Rice University
}

November 2000 


\begin{abstract}
Indivisible units are randomly allocated among agents with a claim / demand on the resources. The available resources fall short of the sum of individual claims.

The proportional method distributes units sequentially, and the probability of receiving a unit at any step is proportional to the unsatisfied claims.

We characterize the family of probabilistic rationing methods meeting the three axioms Consistency, Lower and Upper Composition. It contains the proportional method, all deterministic fixed priority methods, and the priority compositions of proportional methods. The proportional method is the only fair method in the family.
\end{abstract}

Keywords: proportionality, fair division, random allocation, probabilistic rationing.

Hervé Moulin, Rice University, Department of Economics, MS 22, P.O. Box 1892, Houston, TX 77251, USA, moulin @ rice.edu.

\title{
Acknowledgments:
}

Stimulating conversations with Anna Bogomolnaia, Dean Foster, Barry Nalebuff, Yves Sprumont, Richard Stong and Rakesh Vohra are gratefully acknowledged. A preliminary version was presented at the 10th International Conference on Game Theory, SUNY Stony Brook, July 19-23, 1999, and at the Northwestern MEDS Workshop on Microeconomic Theory, July 22-25, 1999.

This research is supported by the NSF under grant SES0096230. 


\section{The Proportional Random Allocation Of Indivisible Units}

\section{Probabilistic rationing methods}

Rationing according to claims is the simplest formal model to date in distributive justice. Each concerned agent holds a claim / demand over the resources, but the resources available fall short of the sum of individual claims. The resources are from a single homogeneous good, and "more is better" for every agent (individual preferences increase in the amount of good received).

The literature on the rationing problem, (starting with Banker [1981], O'Neill [1982], Aumann and Maschler [1985]) assumes an infinitely divisible good (e.g., money), hence individual claims and the amount of available resources are real numbers. Here I look instead at the discrete version of the problem, where the homogeneous good comes in indivisible units, and individual claims / demands, as well as the shares of the resources, are integervalued. Examples include: the allocation of cars of a popular model by GM among its dealers (Cachon and Larivière [1996]); of tickets to a sport event among sponsors, of seats in planes or rooms in hotels among tour operators, of immigration visas among countries (Elster [1992]); of workers to the tasks requested by the different customers; of slots by a network server among users (Demers et al.[1990]), and so on.

Allocating the resources in proportion to individual claims is, without a doubt, the oldest formal rule of distributive justice, dating back to Aristotle. When indivisible units must be allocated, randomization is a time-honored device to achieve fairness. For instance, we can use lotteries to implement the proportionality principle in the sense of expected shares: if we have only one unit to distribute, the probability that a certain agent receives it will be proportional to his own claim.

The simple idea of "expected proportionality" works also when we allocate several units, but it leaves room for many random allocation methods. I argue in this paper that one such method stands out on normative grounds, because it is the only method satisfying a handful of abstract axiomatic requirements. The central concept is that of a probabilistic rationing method: to every (deterministic) integervalued rationing problem (listing an individual claim $x_{i}$ for each agent $i$, and a number $t$ of units to be divided), such a method associates a random allocation of $t$ units among all agents, with the only constraint that agent $i$ cannot receive more than $x_{i}$ units.

One way to construct a probability rationing method when the expected individual shares are given is to minimize the variance of the random variable. Thus if the expected shares at a certain problem $t, x_{i}$ are integers, we split $t$ accordingly, and no lottery is needed (e.g., if $t=5, x_{1}=12, x_{2}=8$, the proportionality principle would force the deterministic shares $y_{1}=3, y_{2}=2$ ). This is not how the method advocated in this paper works. 
Our proportional method is defined as follows: the first unit is randomly allocated to agent $i$ with probability proportional to his initial unsatisfied claim $x_{i}$; the unsatisfied claim of the agent who receives the first unit drops by one; the second unit is randomly allocated in proportion to the unsatisfied claims, and the recipient of the second unit, who may or may not be the same as that of the first, sees his unsatisfied claim drop by one. And so on. The process ends in $t$ steps.

Equivalently, think of the $\sum_{i} x_{i}$ units of claim as labeled by the agents (with $x_{i}$ units labeled by agent $i$ ): the proportional method chooses a random subset of $t$ units of claim, giving equal probability to all subsets.

This probabilistic rationing method gives an expected share to agent $i$ proportional to $x_{i}$. On the other hand, every division of the $t$ units in shares bounded above by individual claims occurs with positive probability (e.g. with $t=5, x_{1}=12, x_{2}=8$, the division $y_{1}=0, y_{2}=5$ occurs with probability $7 / 1938)$.

Our axioms do not impose any restriction on the variance of the allocation rule. They are of two kinds. On the one hand we have three structural invariance properties coming straight out of the classical model (i.e., when the resources and the claims are real-valued):

- Consistency expresses the invariance of the solution when the set of concerned agents changes.

- Lower Composition allows to decompose the allocation of $t$ units into, first, the allocation of $t^{\prime}$ units, $t^{\prime} \leq t$,second, that of $t-t^{\prime}$ units according to the reduced claims.

- Upper Composition considers the two step process where first, $t^{\prime}$ units are allocated, $t^{\prime} \geq t$,and the resulting shares are taken as claims in the second step, where $t$ units are allocated; this two step process is equivalent to the direct allocation of $t$ units.

All three axioms are familiar in the classical rationing model (see Young [1988, 1990], Thomson [1995] and Moulin [1998] [1999]) and their interpretation is identical in the probabilistic model (it is repeated below after Definitions $1,2,3)$. Also preserved from the classical model to the probabilistic one, is an important duality operation exchanging the Lower and Upper Composition axioms.

The other kind of axioms are about fairness, namely the property that the allocation method is not a priori biased for or against certain agents. The most familiar of the fairness axioms-the most ethically transparent as well- is the property known as Equal Treatment of Equals, requiring that two agents with identical claims / demands should receive the same random allocation ex ante. We also discuss some weaker fairness properties.

I offer two types of characterization results. On the one hand the proportional method is the only fair method meeting the three invariance properties discussed three paragraphs ago. Here is one way to put this into a formal statement: the proportional method is characterized by the combination of 4 axioms, Equal Treatment of Equals, Consistency, Lower and Upper Composition. In fact, four characterizations of the proportional method are provided: 
- by Lower and Upper Composition, and Equal Treatment of Equals: Theorem 1 ;

o by Lower Composition and Self-Duality (requiring that the method allocates gains exactly as it allocates losses): Theorem 1;

- by Consistency, Lower and Upper Composition, and Priority-freeness (ruling out absolute priority of any agent over any other; a much weaker requirement than Equal Treatment of Equals): Corollary to Theorem 2;

- by Consistency, Lower Composition and Positive Share (a mild individual rationality statement, insisting that an agent with a positive claim must receive at least one unit with some positive probability; this axiom is not logically related to Equal Treatment of Equals): Theorem 3.

Together, these four characterizations make a strong normative case in favor of the proportional probabilistic rationing method.

On the other hand, the very inequitable priority rationing methods satisfy all the invariance axioms: these deterministic methods work with a fixed ordering of the agents, by giving absolute priority to the claim of the highest agent in the priority ordering, then to that of the next highest priority agent and so on. Hence the next question: besides the proportional and the priority methods, what other asymmetric methods meet all three structural invariance axioms? This leads to our second characterization result, mathematically more complex, and relevant in those contexts where for some exogenous reason, the agents may not or should not be treated symmetrically. Theorem 2 shows that all methods meeting Consistency, Lower and Upper Composition work by, essentially, partitioning the set of concerned agents according to a given preordering, by using the proportional method within each set in the partition (each indifference class of the given preordering), and full priority across indifference classes. The only complication comes from indifference classes containing exactly two agents, for which a one-dimensional family of methods connecting the proportional method to the two priority ones can be used.

- Relation to the literature

The literature on the rationing problem (also called the claim problem) discusses three different models. In the most popular one, that we call the classical model, the good is infinitely divisible $\left(t, x_{i}\right.$ are realvalued) and the allocation is deterministic. When the good comes in indivisible units and the allocation is deterministic, we speak of the discrete model. Finally we refer to the model in this paper, with indivisible goods and random allocations, as the probabilistic model.

The proportional method plays a leading role in the classical model. ${ }^{1}$ There the simplest characterization of the method relies on a single axiom pertaining to transfers of claims/demands across agents: my share is not affected whenever such a transfer occurs between any two other agents. Alternative axioms deal with the merging of two claims into a single claim or the splitting of a single claim into two; see Moulin [1999] for a survey of these (easy) characterization

\footnotetext{
${ }^{1}$ It is undefined in the discrete model, although the choice of an approximation to proportionality has been axiomatically discussed: see Balinski and Shahidi [1997].
} 
results, and Moulin and Stong [2000b] for a discussion of these axioms in the probabilistic model.

A previous paper by this author (Moulin [2000]) introduces the discrete model and establishes a corollary of the current theorem 2: among deterministic methods, only the priority ones satisfy Consistency, Lower and Upper Composition. With the important exception of Sasaki [1996] discussed below, the rest of the literature is entirely devoted to the classical model.

Theorem 3 in Young [1988] is the only characterization result in the classical model that is word for word identical to one of the results in this paper; it is also our most compact characterization of the proportional method, by the combination of Self-Duality and Lower (or Upper) Composition (Statement ii in Theorem 1).

The main result in Moulin [2000] characterizes in the classical model the impact of the four axioms Consistency, Lower and Upper Composition, and Scale Invariance; the latter property has no equivalent in the discrete or probabilistic models. Still the class of methods circumscribed by these four properties is loosely speaking larger than the class described in our Theorem 2. Similarly, if we add Equal Treatment of Equals to the four axioms just listed, we are left with exactly three celebrated methods: the proportional, uniform gains and uniform losses. The latter two equalize respectively the individual net shares and the individual net losses (difference between claim and share).

The two classical methods, uniform gains and uniform losses, can be canonically "extended" to the probabilistic model in the sense that i) the expected shares of the probabilistic method equal the classical deterministic shares and ii) the probabilistic method meets as many of our three invariance axioms as possible. These two methods, called respectively random uniform gains and random uniform losses are discussed in Section 8.

Sasaki [1996] is, to the best of my knowledge, the only prior paper addressing the random allocation of indivisible units according to individual claims: he introduces the random uniform gains method, that he calls the Random Uniform Allocation method, and offers an axiomatic characterization. Sasaki's model, however, is more complicated than our rationing model; it involves allocating the $t$ units of the commodity whether the sum of individual "demands" exceeds $t$ (as required in a rationing model) or falls short of $t$. This model is known as fair division with single-peaked preferences (Sprumont [1991]) and its ingredients are more complex than in the current model: agents are endowed with full fledged preferences over their possible shares. Consequently, the powerful axiom of strategyproofness can be invoked. Sasaki [1996]'s characterization of the random uniform gain method uses three axioms: strategyproofness, efficiency with respect to risk avert Von Neumann-Morgenstern utilities, and anonymity (a familiar strenghtening of Equal Treatment of Equals).

The companion paper Moulin and Stong [2000a] improves upon the current axiomatic results by, essentially, elucidating the full impact of any pair from the three basic axioms Consistency, Lower and Upper Composition. Under mild additional requirements of monotonicity (such as Demand Monotonicity: my ex ante share does not decrease - in the stochastic dominance sense - when my 
demand increases, ceteris paribus) or individual rationality (such as our Positive Shares and Positive Risks requirements: see Section 7) the three families in question are entirely described. Two corollaries characterize the random uniform gains and random uniform losses methods, respectively.

One last piece of literature is relevant to this paper, namely the recent work on cost-sharing methods with heterogeneous goods coming in indivisible units: Moulin [1995], de Nouweland et al. [1995], Sprumont and Wang [1998], Wang [1999]. There a certain cost-sharing method, known as the discrete AumannShapley formula, plays an important role. This method takes a random path from 0 to the (integervalued) vector $x$ of demands, and allocates costs along this path by the marginal contribution principle; all paths have equal probability. Thus the Aumann-Shapley formula obtains, essentially, as the integral of the marginal contributions along the proportional probabilistic rationing method.

- Contents

The model is defined in Section 2, and the proportional method in Section 3. Section 4 introduces the axioms Lower and Upper Composition. Theorem 1 is the object of Section 5. The Consistency axiom is introduced in Section 6 along with the main characterization result (Theorem 2). Section 7 defines the axioms Positive Share and Positive Risk and offers the last two characterizations of the proportional method: Theorem 3. The last Section 8 introduces the canonical probabilistic version of uniform gains and uniform losses; it also defines two more methods of an egalitarian inspiration. These four methods are used to show that our characterization results are tight. The proofs of the most involved results are gathered in the Appendix.

\section{The model}

The set of natural integers is denoted $\ddot{I}=\{0,1,2, \ldots\}$. A rationing method (defined below) is a variable population concept: it can deal with problems involving any finite subset of an exogenously given set $\mathcal{N}$ of potential agents (think of $\mathcal{N}$ as the current population on earth). The set $\mathcal{N}$ can be finite or infinite. A deterministic rationing problem is a triple $(N, t, x)$ where:

- $N$ is a finite subset of $\mathcal{N}$

- $t \in \ddot{I}$ is the number of units to be divided among the agents in $N$

- $x \in \ddot{I}^{N}, x=\left(x_{i}\right)_{i \in N}$, is the profile of claims (demands, liabilities,...) over the resources $t$

- the available resources do not exceed the sum of individual claims: $t \leq x_{N}$ (with the notation $x_{S}=\sum_{s} x_{i}$ )

A deterministic solution to the rationing problem $(N, t, x)$ is an element $z$ of the following set $Z(N, t, x): Z(N, t, x)=\left\{z \in \ddot{I}^{N} \mid 0 \leq z \leq x\right.$ and $\left.z_{N}=t\right\}$.

A probabilistic solution to the rationing problem $(N, t, x)$ is a random variable over $Z(N, t, x)$. As this will cause no confusion, we identify such a random variable with its probability distribution over $Z(N, t, x)$, namely an element $Y$ of the following set $\mathcal{Z}(N, t, x)$ : 


$$
\mathcal{Z}(N, t, x)=\left\{Y: Z(N, t, x) \rightarrow[0,1] \mid \sum_{Z(N, t, x)} Y(z)=1\right\}
$$

A probabilistic rationing method (resp. a deterministic rationing method), is a mapping $\rho$ (resp. $r$ ), associating to every rationing problem $(N, t, x)$ a probabilistic solution $\rho(N, t, x)=Y \in \mathcal{Z}(N, t, x)$ (resp. a deterministic solution $r(N, t, x)=z \in Z(N, t, x))$. Thus for all $z \in Z(N, t, x), \rho(N, t, x)(z)$ is the probability that the $t$ units be allocated as $z$. We denote by $\mathcal{R}$ and $R$ respectively the set of probabilistic and deterministic rationing methods. Clearly, $R \subset \mathcal{R}$.

When the set $\mathcal{N}$ of potential agents may change, we will write a probabilistic rationing method as $\rho(\mathcal{N})$ and their set as $\mathcal{R}(\mathcal{N})$. This notation is useful in Section 6 where we deal with the Consistency axiom. On the other hand the lighter notation $\rho, \mathcal{R}$ is enough most of the time, for instance when we discuss the axioms Lower and Upper Composition that do not involve variations of the sets $\mathcal{N}$ or $N$.

To a probabilistic solution $y$ of the rationing problem $(N, t, x)$, we associate the vector Ey of expected shares:

$$
\forall y \in Z(N, t, x): E y=\sum_{Z(N, t, x)} y(z) z
$$

In general, expected shares are not in $\ddot{I}$, therefore given $\rho$ in $\mathcal{R}$, the mapping $(N, t, x) \rightarrow E \rho(N, t, x)$ is not an element of $R$ (although it selects a deterministic vector of shares). We will nevertheless speak of the expected rationing method $E \rho$ associated with $\rho$.

Our first example is a family of deterministic methods (in $R$ ) called the priority methods. Fix an ordering $\sigma$ of $N$ - i.e., a complete, transitive and asymmetric binary relation on $N-$. The priority method $r^{\sigma}$ works by giving absolute priority to the claim of the first agent in $N$ according to $\sigma$, next (if any resources are left), to the claim of the second agent and so on. Formally, the deterministic solution $r^{\sigma}(N, t, x)=z$ is defined by the following conditions:

$z \in Z(N, t, x)$; for all $i, j \in N:\left\{i\right.$ precedes $j$ in $\sigma$ and $\left.z_{i}<x_{i}\right\} \Rightarrow\left\{z_{j}=0\right\}$.

Priority methods are as unfair as can be, but fairness can be restored by the unbiased randomization of the ordering $\sigma$. Formally, the random priority method $r p$ is defined as follows: for any problem $(N, t, x)$, and any $z \in Z(N, t, x)$,denote by $O(z)$ the set of all orderings $\sigma$ of $N$ such that $r^{\sigma}(N, t, x)=z$. Then

$$
r p(N, t, x)(z)=\frac{|O(z)|}{|N| !} \text { for all } z \in Z(N, t, x)
$$


(where $|A|$ denotes the cardinality of $A$, and $|\emptyset|=0$ ).

This defines a method $r p$ in $\mathcal{R}$; this method is fair in the sense of the Equal Treatment of Equals property: two agents with identical claims are treated identically by the method. Formally,

$$
\left\{x_{i}=x_{j}\right\} \Longrightarrow \rho_{i}(N, t, x)=\rho_{j}(N, t, x)
$$

where $\rho_{i}(N, t, x)$ is the projection on the $i$-th coordinate of the random variable $\rho(N, t, x)$ - the $i$-th marginal of the probability distribution over $Z(N, t, x)$ -2 .

We conclude this section by defining the duality operator which plays an important role in this version of the rationing problem, as it does in the more familiar model with deterministic and real-valued solutions (see Moulin [1999]). The dual of a rationing method allocates losses in the same way as the primal method allocates gains (and vice-versa). Given $\rho$ in $\mathcal{R}$, its dual $\rho^{*}$ is defined as:

$$
\rho^{*}(N, t, x)=x-\rho\left(N, x_{N}-t, x\right) \text { for all } N, t, x .
$$

In the above formula, $y=\rho\left(N, x_{N}-t, x\right)$ is a random variable with range in $Z\left(N, x_{N}-t, x\right)$, and $y^{*}=\rho^{*}(N, t, x)$ is a random variable in $Z(N, t, x)$. Equivalently, the probability distributions of $y$ and $y^{*}$ are related as follows:

$$
\rho^{*}(N, t, x)(z)=\rho\left(N, x_{N}-t, x\right)(x-z) \text { for all } N, t, x \text { and } z \in Z(N, t, x)
$$

If $r$ is a deterministic method, so is its dual $r^{*}$. The duality operator commutes with the computation of expected shares: $E \rho^{*}(N, t, x)=x-E \rho\left(N, x_{N}-\right.$ $t, x)$ which can be written as $E \rho^{*}=(E \rho)^{*}$ if we think of $E \rho$ as a (real valued) rationing method.

The dual of the priority method $r^{\sigma}$ is $r^{\sigma^{*}}$, where $\sigma^{*}$ is the symmetric ordering of $\sigma$. Therefore the random priority method $r p$ is self-dual: $r p^{*}=r p$.

\section{The proportional method}

This is the central object of interest in this paper. The simplest definition of the proportional method is recursive, and relies on the stochastic filling or emptying of an urn.

Fix $N$ and a profile of claims $x$. Fill an urn with $x_{N}$ balls, $x_{i}$ of which are of color $i$. Empty the urn one ball at a time and without replacement, drawing at each step each ball left in the urn with equal probability. After $x_{N}-t$ balls have been removed in this way, the (random) content of the urn is $Y=\operatorname{pro}(N, t, x)$.

\footnotetext{
${ }^{2}$ The expected shares of the random priority method are a familiar solution in the literature on deterministic, real-valued rationing problems: $\operatorname{Erp}(N, t, x)$ is the Shapley value of the cooperative game $V(S)=\min \left\{t, x_{S}\right\}$ for all $S \subseteq N$ (see O'Neill [1982], Aumann and Maschler [1985]).
} 
Dually, start from an empty urn and add one ball at a time. The color of the first ball is $i$ with probability $x_{i} / x_{N}$. The profile of claims is adjusted by substracting one unit from $x_{i}$ if a ball of color $i$ is drawn, and we repeat the process, drawing a second ball of color $j$ with probability proportional to $j^{\prime} s$ remaining claim, substracting one from the claim of the winner, and so on. After $t$ balls have been placed in the urn in this fashion, its (random) content is $Y=\operatorname{pro}(N, t, x)$.

We give now the proportional method in closed form, namely we compute the probability distribution of $\operatorname{pro}(N, t, x)$.

Given $N$, a finite subset of $\mathcal{N}$, and $x$ in $\ddot{I}^{N}$, we denote by $D(N, x)$ the set of monotonic paths from 0 to $x$. With the notation $[0, x]$ for the subset of $\ddot{I}^{N}$ defined by $0 \leq z \leq x$, a generic element $d$ of $D(N, x)$ is a mapping from $\left\{0,1, \ldots, x_{N}\right\}$ into $[0, x]$ such that:

$$
d_{N}(t)=t \text { and } t \leq t^{\prime} \Longrightarrow d(t) \leq d\left(t^{\prime}\right) \text { for all } t, t^{\prime} \text { in }\left\{0,1, \ldots x_{N}\right\}
$$

(in particular, $d(0)=0$ and $d\left(x_{N}\right)=x$ ). Note that by choosing a monotonic path $d$ for each choice of $(N, x)$, we obtain a deterministic rationing method (an element of $R$ ). All methods in $R$ satisfying the monotonicity property: $t \leq t^{\prime} \Longrightarrow r(N, t, x) \leq r\left(N, t^{\prime}, x\right)$, can be obtained in this fashion ${ }^{3}$.

In the random urn filling algorithm described two paragraphs earlier, we compute the probability that the successive draws generate a certain path $d$ in $D(N, x)$. Denote by $\left\{i_{1}, i_{2}, \ldots, i_{x_{N}}\right\}$ the sequence of successive increments of $d$ :

$$
d(t)-d(t-1)=e_{i_{t}} \text { for } t=1,2, \ldots, x_{N}
$$

(where $e_{j}$ is the $j-t h$ coordinate vector).

Because agent $i$ appears exactly $x_{i}$ times in the sequence $\left\{i_{1}, i_{2}, \ldots\right\}$, the probability of $d$ is easily computed:

$$
\operatorname{pro}(d)=\frac{\prod_{i \in N} x_{i} !}{x_{N} !} \text { for all } d \in D(N, x)
$$

The important fact is that all paths $d$ in $D(N, x)$ are equiprobable. Therefore the probability pro $(N, t, x)(z)$ is the number of paths in $D(N, x)$ going through $z$, divided by the cardinality of $D(N, x)$. Denoting by $\delta(N, x)$ the latter cardinality (namely $\delta(N, x)=x_{N} ! / \prod_{i \in N} x_{i} !$ ), we obtain the desired closed form of the proportional method:

$$
\operatorname{pro}(N, t, x)(z)=\frac{\delta(N, z) \delta(N, x-z)}{\delta(N, x)}=\frac{t !\left(x_{N}-t\right) !}{x_{N} !} \prod_{i \in N} \frac{x_{i} !}{z_{i} !\left(x_{i-} z_{i}\right) !}
$$

\footnotetext{
${ }^{3}$ For instance, the priority method $r^{\sigma}$ corresponds to a monotonic path following the edges of the box $[0, x]$.
} 
The above computation can be repeated for the urn-emptying algorithm. The probability that the successive draws from the urn generate a given path $d$ is again independent of $d$, and equal to $1 / \delta(n, x)$. This proves that the two algorithms, emptying or filling, generate the same probabilistic rationing method and that this method is self-dual. We omit the straightforward details.

We check finally that the expected shares of the proportional method are, indeed, proportional to the claims:

$$
\operatorname{Epro}(N, t, x)=\frac{t}{x_{N}} x \quad \text { for } \text { all } N, t, x
$$

Given a profile of claims $x$, we consider the urn-emptying algorithm starting from an urn with $x_{N}$ balls, among which $x_{i}$ are of color $i$, for all $i \in N$. After $k$ balls have been randomly extracted from the urn, all subsets of $\left(x_{N}-k\right)$ balls (from the $x_{N}$ initial balls) have the same probability of being left in the urn. Therefore the expected number of $i$-balls left in the urn is proportional to $x_{i}$, for all $i$. Equation (5) follows at once.

\section{Lower and Upper Composition}

In the recursive definitions of the proportional method by the urn-filling or emptying sequences, a key feature is that the allocation of successive gains or losses is history independent: to add the $k-t h$ ball in the urn-filling sequence, we only need to know the current unsatisfied claim of each agent (i.e., initial claim minus balls allocated to this agent in the first $(k-1)$ steps); it does not matter what the initial claims were, or who has been lucky or unlucky during the allocation of the first $(k-1)$ balls. Symmetrically, in the urn-emptying algorithm, the state variable is the remaining content of the urn, no other detail of the first $k-1$ draws matters. These two properties are the object of our first two axioms.

\section{Definition 1}

The probabilistic rationing method $\rho$ in $\mathcal{R}$ has the Lower Composition property (in short, $L C$ ), if for any problem $(N, t, x)$ and any $t^{\prime}, 0 \leq t^{\prime} \leq t$, the random allocation $\rho(t, x)$ is the sum of two random allocations as follows. First allocate $t^{\prime}$ units according to $\rho\left(t^{\prime}, x\right)$ with realization $z^{\prime}$; next allocate $t-t^{\prime}$ units according to $\rho\left(t-t^{\prime}, x-z^{\prime}\right)$ :

$$
\rho(t, x)={ }^{1} \rho\left(t^{\prime}, x\right)+{ }^{2} \rho\left(t-t^{\prime}, x-{ }^{1} \rho\left(t^{\prime}, x\right)\right)
$$

where the variables ${ }^{1} \rho$ and ${ }^{2} \rho$ are stochastically independent. Equation (6) is written equivalently as:

$$
\rho(t, x)(z)=\sum_{z^{\prime} \in Z\left(t^{\prime}, z\right)} \rho\left(t^{\prime}, x\right)\left(z^{\prime}\right) \cdot \rho\left(t-t^{\prime}, x-z^{\prime}\right)\left(z-z^{\prime}\right) \text { for all } z \in Z(N, t, x)
$$


If $\rho=r$ is a deterministic rationing method, $r \in R$, and we set ${ }^{1} \rho={ }^{2} \rho=r$ in equation (6), we obtain the property also called Lower Composition in Moulin [2000] [1999] (Young [1988] calls the axiom Composition). The interpretation of LC in that context carries over to our probabilistic model: if we know a lower bound $t^{\prime}$ of the resources $t$ that will ultimately be available, we may (randomly) allocate $t^{\prime}$ units now, keep track only of the unsatisfied claims and address later the (smaller) problem of allocating the remaining $t-t^{\prime}$ units.

\section{Definition 2}

The probabilistic rationing method $\rho$ in $\mathcal{R}$ has the Upper Composition property (in short $U C$ ) if for any problem $(N, t, x)$ and any $t^{\prime}, t \leq t^{\prime} \leq x_{N}$, the random allocation $\rho(t, x)$ can be decomposed in the following two random allocations: allocate first $t^{\prime}$ units according to $\rho\left(t^{\prime}, x\right)$ with realization $z^{\prime}$; next allocate $t$ units according to $\rho\left(t, z^{\prime}\right)$ :

$$
\rho(t, x)={ }^{2} \rho\left(t,{ }^{1} \rho\left(t^{\prime}, x\right)\right)
$$

where the variables ${ }^{1} \rho,{ }^{2} \rho$ are stochastically independent. Equivalently:

$$
\rho(t, x)(z)=\sum_{z^{\prime} \in Z\left(t^{\prime}, x\right), z \leq z^{\prime}} \rho\left(t^{\prime}, x\right)\left(z^{\prime}\right) \cdot \rho\left(t, z^{\prime}\right)(z) \text { for all } z \in Z(N, t, x)
$$

As above, the property (8) applied to a deterministic method $r \in R, r=$ $\rho={ }^{1} \rho={ }^{2} \rho$, is also called Upper Composition in Moulin [2000] [1999], and Path Independence in Moulin [1987], Herrero [1998] and others. The standard interpretation carries over: if we know an upper bound $t^{\prime}$ of the actual resources $t$, we may use our method to (randomly) reduce the claims at the level $\rho\left(t^{\prime}, x\right)$ (erasing $x$ from the records) and deal later with the allocation of the $t$ units according to the reduced claims; the latter problem involves a smaller loss than the initial problem.

The definition of the proportional method by the urn-filling or emptying algorithms makes it clear that this method meets both Lower and Upper Composition.

Our next result is a useful representation of all methods in $\mathcal{R}$ meeting LC or UC; it also establishes that these two axioms are dual of each other. We use the following notation. For any method $\rho$ in $\mathcal{R}$ and any $(N, x)$ such that $x_{N}>0$ we set :

$$
\alpha(N, x)=\rho(N, 1, x) ; \beta(N, x)=x-\rho\left(N, x_{N}-1, x\right)
$$

We call $\alpha$ (resp. $\beta$ ) the incremental gain (resp. incremental loss) of the method $\rho$. Thus $\alpha(N, x)$ is in $\mathcal{Z}(N, 1, x)$, namely a random variable on the set of unit vectors $e^{i}$ such that $x_{i}>0$ : it allocates a single unit when the claims are $x$. Dually, $\beta(N, x)$ allocates one unit of deficit among the agents with a positive claim: $\beta(N, x)$ is in $x-\mathcal{Z}\left(N, x_{N}-1, x\right)=\mathcal{Z}(N, 1, x)$ as well. 
Note that the definition of $\beta$ is equivalently written as :

$$
\beta(N, x)\left(e^{i}\right)=\rho\left(N, x_{N}-1, x\right)\left(x-e^{i}\right) \text { for all } i \text { s.t. } x_{i}>0
$$

Lemma 1

i) Given a method $\rho$ in $\mathcal{R}$ and the dual method $\rho^{*}$, the incremental gain (resp. loss) of $\rho$ equals the incremental loss (resp. gain) of $\rho^{*}: \beta^{*}=\alpha$ and $\alpha^{*}=\beta$.

ii) The properties Lower and Upper Composition are dual of each other.

iii) The method $\rho$ in $\mathcal{R}$ meets Lower Composition if and only if it is generated from its incremental gain $\alpha$ along the following increasing random path:

$$
\begin{aligned}
\delta^{+}(0, x) & =0 ; \delta^{+}(1, x)=\alpha(x) \\
\delta^{+}(k+1, x) & =\delta^{+}(k, x)+\alpha\left(x-\delta^{+}(k, x)\right) \text { for } k=0,1, \ldots, t-1 \\
\rho(t, x) & =\delta^{+}(t, x)
\end{aligned}
$$

or equivalently:

$$
\rho(t, x)(z)=\sum_{d \in D(z)} \prod_{k=0}^{t-1} \alpha(x-d(k))(d(k+1)-d(k)) \text { for all } z \in Z(N, t, x)
$$

iv) The method $\rho$ in $\mathcal{R}$ meets Upper Composition if and only if it is generated from its incremental loss $\beta$ along the following decreasing random path:

$$
\begin{aligned}
\delta^{-}\left(x_{N}, x\right) & =x ; \delta^{-}\left(x_{N}-1, x\right)=x-\beta(x) \\
\delta^{-}(k-1, x) & =\delta^{-}(k, x)-\beta\left(\delta^{-}(k, x)\right) \text { for } k=x_{N}, x_{N}-1, \ldots, t+1 \\
\rho(t, x) & =\delta^{-}(t, x)
\end{aligned}
$$

or equivalently:

$$
\rho(t, x)(z)=\sum_{d \in D(x-z)} \prod_{k=1}^{x_{N}-t} \beta(z+d(k)) .(d(k)-d(k-1)) \text { for all } z \in Z(N, t, x)
$$

Proof

Statement i is clear. For statement ii, start with $\rho$ in $\mathcal{R}$ meeting LC, and check that $\rho^{*}$ meets UC. We fix $N, x$ and two integers $s, s^{\prime}$ s.t. $s \leq s^{\prime} \leq x_{N}$ and will show equation (8) namely:

$$
\rho^{*}(s, x)={ }^{2} \rho^{*}\left(s,{ }^{1} \rho^{*}\left(s^{\prime}, x\right)\right)
$$


Set $t=x_{N}-s, t^{\prime}=x_{N}-s^{\prime}$ and develop the right-hand term as:

$$
\begin{aligned}
{ }^{1} \rho^{*}\left(s^{\prime}, x\right)-{ }^{2} \rho\left(s^{\prime}-s,{ }^{1} \rho^{*}\left(s^{\prime}, x\right)\right) & =\left(x-{ }^{1} \rho\left(t^{\prime}, x\right)\right)-{ }^{2} \rho\left(t-t^{\prime}, x-{ }^{1} \rho\left(t^{\prime}, x\right)\right) \\
& =x-\left({ }^{1} \rho\left(t^{\prime}, x\right)+{ }^{2} \rho\left(t-t^{\prime}, x-{ }^{1} \rho\left(t^{\prime}, x\right)\right)\right) \\
& =x-\rho(t, x)=\rho^{*}(s, x)
\end{aligned}
$$

as was to be proven.

For statement iii, start with $\rho$ in $\mathcal{R}$ meeting LC. Fix $N, x$ and check by induction on $t$ that $\rho(t, x)$ obtains as (12). This is clear for $t=1$. For an arbitrary $t$, apply (6) to $t^{\prime}=t-1$ and use the induction assumption.

Conversely, assume that $\rho$ is constructed from its incremental gain via the random path (12). Checking property (6) is straightforward.

Finally, statement iv. Start with $\rho$ in $\mathcal{R}$ meeting UC. Thus (by statements ii and iii) $\rho^{*}$ meets $\mathrm{LC}$ and $\rho^{*}$ obtains from its incremental gain variable $\alpha^{*}$ via the increasing random path $\delta^{+}$given by (12).

Given $(N, t, x)$, we define a decreasing random path $\delta^{-}$as follows:

$$
\delta^{-}(k, x)=x-\delta^{+}\left(x_{N}-k, x\right) \text { for } k=x_{N}, x_{N}-1, \ldots, t
$$

Compute next

$$
\begin{aligned}
\delta^{-}(k-1, x) & =x-\delta^{+}\left(x_{N}-k+1, x\right) \\
& =x-\delta^{+}\left(x_{N}-k, x\right)-\alpha^{*}\left(x-\delta^{+}\left(x_{N}-k, x\right)\right) \\
& =\delta^{-}(k, x)-\alpha^{*}\left(\delta^{-}(k, x)\right)=\delta^{-}(k, x)-\beta\left(\delta^{-}(k, x)\right)
\end{aligned}
$$

(where $\alpha^{*}=\beta$ follows from statement i). By construction $\delta^{-}\left(x_{N}, x\right)=x$ and $\delta^{-}(t, x)=x-\rho^{*}\left(x_{N}-t, x\right)=\rho(t, x)$ and the proof is complete.

A priority method $r^{\sigma}$ meets the (deterministic) properties LC and UC. Its (deterministic) incremental gain and loss are:

$$
\begin{aligned}
& \alpha(N, x)=e^{i} \text { where } i \text { maximizes } \sigma \text { in } M(N, x) \\
& \beta(N, x)=e^{j} \text { where } j \text { minimizes } \sigma \text { in } M(N, x)
\end{aligned}
$$

(recall the notation $M(N, x)=\left\{i \in N \mid x_{i}>0\right\}$ )

However, any strict convex combination of priority methods fails both axioms. We check this for the random priority method (1) and refer the reader to Remark 3 (see Appendix, after proof of Lemma 4) where we explain the interesting fact that LC, UC are almost never stable by convex combinations.

Consider $N=\{1,2\}$, and $\left(x_{1}, x_{2}\right)$ such that $x_{i} \geq 2, i=1,2$. The variable $r p(N, 2, x)$ takes the value $(2,0)$ or $(0,2)$, each with probability $\frac{1}{2}$; it never reaches $(1,1)$. On the other hand ${ }^{1} \rho(1, x)+{ }^{2} \rho\left(1, x-{ }^{1} \rho(1, x)\right)$ takes the value $(2,0),(1,1)$ and $(0,2)$ with probability $\frac{1}{4}, \frac{1}{2}$ and $\frac{1}{4}$, respectively. 
Of course, the incremental gain and loss of the proportional method are :

$$
\alpha(N, x)\left(e^{i}\right)=\beta(N, x)\left(e^{i}\right)=\frac{x_{i}}{x_{N}} \text { for all } i
$$

\section{First characterization of the proportional method}

\section{Theorem 1}

The proportional probabilistic rationing method is characterized by the two following combinations of axioms:

i) Lower Composition, Upper Composition, and Equal Treatment of Equals,

ii) Self-duality and Lower (or Upper) Composition.

In the classical model (deterministic shares, divisible goods), there is a large family of methods meeting the three properties in statement $i$ : it includes uniform gains, uniform losses, as well as asymmetric versions of these methods. On the other hand, statement ii holds true word for word in the classical model: Theorem 3 in Young [1988]. Note that self-duality is an invariance property of which the normative content is hard to interpret: Aumann and Maschler [1985] proposes a bargaining interpretation of the axiom.

\section{Proof}

Statement $i$

We already know that the method pro meets the three axioms listed. Conversely, let $\rho$ be a method in $\mathcal{R}$ meeting LC, UC and ETE. Its incremental gain and loss distributions are denoted $\alpha, \beta$ and we use the simpler notations $\alpha_{i}(x)=\beta_{i}(x)$ in lieu of $\alpha(N, x)\left(e^{i}\right), \beta(N, x)\left(e^{i}\right)$. If $x_{i}=0(i \notin M(N, x))$ we posit $\alpha_{i}(x)=\beta_{i}(x)=0$ (the earlier definition requires $x_{i}>0$ ).

For any $x$ in $\ddot{I}^{N}$ s.t. $x_{N} \geq 3$, we apply (7) (namely, LC) to $t^{\prime}=1, t=x_{N}-1$ and an agent $i \in M(x)$ :

$$
\rho\left(x_{N}-1, x\right)\left(x-e^{i}\right)=\sum_{j \in M(x)} \alpha_{j}(x) \cdot \rho\left(x_{N}-2, x-e^{j}\right)\left(x-e^{i j}\right)
$$

where for $j \neq i$, the notation $e^{i j}$ stands for $e^{i}+e^{j}$ and $e^{i i}$ means $2 e^{i}$. Notice that if $x_{i}=1$, the vector $x-e^{i i}$ has a negative coordinate but in this case there is no term $i$ in the right-hand sum and we set $\rho\left(x_{N}-2, x-e^{j}\right)\left(x-e^{i i}\right)$ equal to zero: this is equivalent to our convention $\beta_{i}(z)=0$ whenever $z_{i}=0$.

Now the above equation writes

$$
\beta_{i}(x)=\sum_{j \in M(x)} \alpha_{j}(x) \cdot \beta_{i}\left(x-e^{j}\right) \text { for all } x \text { s.t. } x_{N} \geq 3 \text { and all } i \in M(x)
$$

Dually, we apply (9) (namely, UC) to $t=1, t^{\prime}=x_{N}-1$ and we obtain

$$
\alpha_{i}(x)=\sum_{j \in M(x)} \beta_{j}(x) \cdot \alpha_{i}\left(x-e^{j}\right) \text { for all } x \text { s.t. } x_{N} \geq 3 \text { and all } i \in M(x)
$$


We use the system (16) (17) to show, by induction on $x_{N}$, that $\alpha=\beta$ is the proportional incremental distribution. If $x$ is a unit vector $\left(x_{N}=1\right)$ there is nothing to prove, next consider the case $x_{N}=2$. If $x=2 e^{i}$, there is nothing to prove; we are left with the case $x=e^{1}+e^{2}$. We cannot apply LC as above because $x_{N}-1=1$, but ETE gives $\alpha_{i}(x)=\frac{1}{2}$ and $\beta_{i}(x)=\frac{1}{2}$ for $i=1,2$, as desired. Note that this is the only place in the proof where we use the equity assumption.

Next we assume the property holds up to $k \geq 2$, and pick a vector $x$ with $x_{N}=k+1$. We can apply (16) hence for all $i \in M(x)$ :

$$
\beta_{i}(x)=\alpha_{i}(x) \cdot \frac{x_{i}-1}{x_{N}-1}+\sum_{M(x) \backslash i} \alpha_{j}(x) \cdot \frac{x_{i}}{x_{N}-1}
$$

Upon rearranging and using $\alpha_{M(x)}=1$, this gives:

$$
\beta_{i}(x)+\frac{1}{x_{N}-1} \cdot \alpha_{i}(x)=\frac{x_{i}}{x_{N}-1}
$$

Similarly, developing (17) with the help of the inductive assumption:

$$
\alpha_{i}(x)+\frac{1}{x_{N}-1} \cdot \beta_{i}(x)=\frac{x_{i}}{x_{N}-1}
$$

For all $i \in M(x)$, the above two equations form a non singular system in $\alpha_{i}(x), \beta_{i}(x)$ (because $x_{N}-1 \geq 2$ ), with solution $\alpha_{i}(x)=\beta_{i}(x)=x_{i} / x_{N}$ as desired.

Statement ii

Now we assume that $\rho$ is self-dual and meets LC, UC. The previous proof can be entirely replicated, provided we show that for a vector $x=e^{1}+e^{2}$, we have $\alpha_{i}(x)=\beta_{i}(x)=\frac{1}{2}$ for $i=1,2$. Observe that for any method $\rho$ in $\mathcal{R}$, the definition of $\alpha, \beta,(10)$ (11) imply for such a vector $x$ :

$$
\alpha_{1}(x)=\beta_{2}(x) ; \alpha_{2}(x)=\beta_{1}(x)
$$

By Lemma 1 and self-duality, $\alpha=\beta$, and the proof is complete.

\section{Remark 1}

The proof shows we only need to assume Equal Treatment of Equals for the problems $(N, 1, x)$ where two agents claim 1 unit each and all other claims are zero. This is arguably a mild symmetry property.

The natural next step is to characterize all methods, symmetric or not, satisfying Lower and Upper Composition: if we drop the ETE requirement, all (deterministic) priority methods $r^{\sigma}$ are now in the family captured by the combination LC cum UC. What else? 
The complete answer to this question for any cardinality of $\mathcal{N}$ is quite complicated: see Moulin and Stong [2000a]. In the current paper, we answer the question only in the case $|\mathcal{N}|=2$ : this turns out to be enough for our main result, Theorem 2 in the next section. For the rest of the section, we assume $\mathcal{N}=N=\{1,2\}$.

For any real number $\lambda$, we write $\mu=1-\lambda$; for any $\lambda$ such that $0<\lambda<1$ and $\lambda \neq \frac{1}{2}$, we define an incremental gain and an incremental loss distributions:

$$
\alpha_{1}^{\lambda}(x)=\frac{\left(\lambda^{x_{1}}-\mu^{x_{1}}\right) \cdot \mu^{x_{2}}}{\lambda^{x_{1}+x_{2}}-\mu^{x_{1}+x_{2}}} ; \alpha_{2}^{\lambda}(x)=\frac{\lambda^{x_{1}} \cdot\left(\lambda^{x_{2}}-\mu^{x_{2}}\right)}{\lambda^{x_{1}+x_{2}}-\mu^{x_{1}+x_{2}}} \text { for all } x \text { s.t. } x_{1}+x_{2}>0
$$

$$
\beta_{1}^{\lambda}(x)=\frac{\left(\lambda^{x_{1}}-\mu^{x_{1}}\right) \cdot \lambda^{x_{2}}}{\lambda^{x_{1}+x_{2}}-\mu^{x_{1}+x_{2}}} ; \beta_{2}^{\lambda}(x)=\frac{\mu^{x_{1}} \cdot\left(\lambda^{x_{2}}-\mu^{x_{2}}\right)}{\lambda^{x_{1}+x_{2}}-\mu^{x_{1}+x_{2}}} \text { for all } x \text { s.t. } x_{1}+x_{2}>0
$$

Clearly $\alpha^{\lambda}(x)$ and $\beta^{\lambda}(x)$ are two probability distributions on $M(x)$. Note the simpler notation $\alpha_{i}(x)$ instead of $\alpha(x)\left(e^{i}\right)$.

\section{Lemma 2}

For all $\lambda$ such that $0<\lambda<1$ and $\lambda \neq \frac{1}{2}$, there is a unique probabilistic rationing method, denoted $\rho^{\lambda}$, that satisfies Lower and Upper Composition, has $\alpha^{\lambda}$ for incremental gain distribution, and $\beta^{\lambda}$ for incremental loss distribution.

From Lemma 1, there is exactly one method in $\mathcal{R}$ meeting LC and with incremental gain $\alpha^{\lambda}$; similarly there is a unique method meeting $\mathrm{UC}$ and with incremental loss $\beta^{\lambda}$. Lemma 2 states that these two methods coincide. Its proof is in the Appendix, along with that of Lemma 3 below.

Note that, as $\lambda$ goes to zero, the probabilistic rationing method $\rho^{\lambda}$ converges to the priority method $r^{\sigma}$ where agent 1 has priority over agent 2 . Here "convergence" is pointwise, namely for any given $t$ and $x$. Similarly, $\rho^{\lambda}$ converges to the dual priority method $r^{\sigma^{*}}$ when $\lambda$ goes to one. Finally, as $\lambda$ goes to $\frac{1}{2}, \rho^{\lambda}$ converges to the proportional method.

Therefore we speak of the continuous parametric family $\rho^{\lambda}$ in $\mathcal{R}$, where $\lambda$ varies in $[0,1]$, where $\rho^{0}, \rho^{1}$ are the two priority methods, and $\rho^{\frac{1}{2}}$ is the proportional one. In this family, duality is the symmetry around $\frac{1}{2}$, namely $\left(\rho^{\lambda}\right)^{*}=\rho^{\mu}$. In particular, the proportional method is the only self-dual method.

Lemma 3

The probabilistic rationing methods $\rho^{\lambda}, 0 \leq \lambda \leq 1$, are the only methods in $\mathcal{R}(12)$ meeting both Lower and Upper Composition. 


\section{Consistency, and the main characterization result}

The Consistency property (in short, CSY), relates the solutions of rationing problems with different "populations", i.e., different sets of agents sharing the resources. In a nutshell, this extremely popular axiomatic requirement requires the following: if the method applied to population $N$ selects a certain allocation of the resources, the method applied to a subset $N^{\prime}$ of $N$ and to the total resources received by $N^{\prime}$ in the $N$ - allocation, must confirm the shares of these agents.

One way to write the definition of the CSY axiom for a method in $\mathcal{R}(\mathcal{N})$ makes it formally identical to the usual definition for deterministic methods:

$$
\rho_{-i}(N, t, x)={ }^{2} \rho\left(N \backslash i, t-{ }^{1} \rho_{i}(N, t, x), x_{-i}\right) \text { for all } N, t, x \text { and } i
$$

In equation (20), the left hand term is the projection of the random variable $\rho(N, t, x)$ onto $N \backslash i$ (that is, we ignore the $i-t h$ coordinate). The right hand side is the composition of two stochastically independent random variables: first ${ }^{1} \rho_{i}$ determines agents $i^{\prime} s$ (random) share when $t$ is divided among $N$; for every realization $z_{i}$ of this share, the random variable ${ }^{2} \rho$ determines the allocation of $t-z_{i}$ among $N \backslash i$.

Thus (20) incorporates the random character of the allocation process in the standard formulation of Consistency. In order to divide $t$ units among the agents in $N$, we may, for any agent $i$ in $N$, separate the allocation to agent $i$ from that to the agents in $N \backslash i$ : first, we randomly select agent $i^{\prime} s$ share $z_{i}$ in the $N$-rationing problem, next we randomly allocate the remaining $t-z_{i}$ units among $N \backslash i$. Each of the two steps follows the probability distribution selected by the rationing method; the key feature is that these two steps are stochastically independent. The CSY axiom requires that the two steps allocation process just described and the one step allocation of $t$ units among $N$ be equivalent, in the sense of generating precisely the same two marginal probability distributions for the share $Y_{i}$ of agent $i$, and the same two marginal probability distributions for the vector of shares $Y_{[N \backslash i]}$ of the agents other than $i$.

Next we give the official definition of CSY in terms of the probability distribution induced by $\rho$. We introduce a piece of notation first. Given $N, t, x, i \in N$ and $z_{i}, 0 \leq z_{i} \leq t$, we write

$$
\rho(N, t, x)\left(Y_{i}=z_{i}\right)=\rho(N, t, x)\left(\left\{z_{i}\right\} \times Z\left(N \backslash i, t-z_{i}, x_{-i}\right)\right)
$$

This is the probability that at the rationing problem $(N, t, x)$, agent $i$ receives $z_{i}$ : the notation uses the random variable $Y_{i}$ for clarity only.

Definition 3

The probabilistic rationing method $\rho, \rho \in \mathcal{R}(\mathcal{N})$, satisfies Consistency if for any $N, t, x$ and $i$ in $N$ we have: 
$\rho(N, t, x)(z)=\rho\left(N \backslash i, t-z_{i}, x_{-i}\right)\left(z_{-i}\right) \cdot \rho(N, t, x)\left(Y_{i}=z_{i}\right)$ for all $z \in Z(N, t, x)$

We note that property (21) is vacuously true if $|N|=2$ therefore the Consistency axiom has no bite unless $|\mathcal{N}| \leq 3$. Another standard feature of CSY is self-duality: a method in $\mathcal{R}(\mathcal{N})$ meets CSY if and only if its dual method does (proof is straightforward).

Lemma 4

A priority method $r^{\sigma}$ meets Consistency; the random priority method does not. The proportional method meets Consistency as well.

Proof

That a priority method is Consistent is both clear and well known (Moulin [2000]). To check that random priority is not Consistent, we consider formula (21) for $N=\{1,2,3\}, t=2$, and $x=(2,1,1)$. Compute first the probability distribution of $\operatorname{rp}(N, 2,(2,1,1))$ :

$$
\begin{aligned}
& (2,0,0) \text { and }(0,1,1) \text { each with proba. } .^{1} / 3 \\
& (1,1,0) \text { and }(1,0,1) \text { each with proba. } .^{1} / 6
\end{aligned}
$$

Thus agent 3 gets 1 or 0 unit, each with proba ${ }^{1 / 2}$. Say agent 3 gets 1 unit; the $r p$ method among $N \backslash 3$ allocates the remaining unit to either agent 1 or 2 with equal probability. Hence for $z=(1,0,1)$ the right-hand term in $(21)$ is $(1 / 2) \cdot(1 / 2)=1 / 4$ whereas the left hand term is $1 / 6$. Equation (21) fails similarly for every $z$ in $Z(N, 2,(2,1,1))$.

We check next that the proportional method meets Consistency. Fix $N, t, x$, $i$ and $z$ as in (21) and compute from (4):

$$
\operatorname{pro}(N, t, x)\left(Y_{i}=z_{i}\right)=\frac{t !\left(x_{N}-t\right) !}{x_{N} !} \cdot \frac{x_{i} !}{z_{i} !\left(x_{i}-z_{i}\right) !} \sum \prod_{N \backslash i} \frac{x_{j} !}{y_{j} !\left(x_{j}-y_{j}\right) !}
$$

where the sum bears on all $y_{-i}$ in $Z\left(N \backslash i, t-z_{i}, x_{-i}\right)$. Use (4) again to write $\operatorname{pro}\left(N \backslash i, t-z_{i}, x_{-i}\right)\left(Z\left(N \backslash i, t-z_{i}, x_{-i}\right)\right)=1$ :

$$
\frac{\left(t-z_{i}\right) !\left(x_{N \backslash i}+z_{i}-t\right) !}{x_{N \backslash i} !} \sum \prod_{N \backslash i} \frac{x_{j} !}{y_{j} !\left(x_{j}-y_{j}\right) !}=1
$$

Apply (4) one more time:

$$
\operatorname{pro}\left(N \backslash i, t-z_{i}, x_{-i}\right)\left(z_{-i}\right)=\frac{\left(t-z_{i}\right) !\left(x_{N \backslash i}+z_{i}-t\right) !}{x_{N \backslash i} !} \prod_{N \backslash i} \frac{x_{j} !}{z_{j} !\left(x_{j}-z_{j}\right) !}
$$


Multiplying the three equations above (the product of the three left-hand terms equals that of the three right-hand terms) and simplifying yields the desired conclusion.

Our main result characterizes all the methods in $\mathcal{R}(\mathcal{N})$ meeting the three properties CSY, LC and UC when $\mathcal{N}$ contains three or more agents. From the examples discussed so far, only the priority methods $r^{\sigma}$ and the proportional method pro successfully pass these three tests when $|\mathcal{N}| \geq 3$. Moreover the methods $\rho^{\lambda}, 0<\lambda<1$, in Theorem 1 pass the three tests when $|\mathcal{N}|=2$ (CSY being vacuous). The entire family delineated by our three axioms is made of simple combinations of these methods.

Definition 4

Given $\mathcal{N}$, a method $\rho$ in $\mathcal{R}(\mathcal{N})$ and two agents $i, j$ in $\mathcal{N}$, we say that $\rho$ gives priority to $i$ over $j$ if for all $N, t, x$ we have

$$
\left\{\rho(N, t, x)(z)>0 \text { and } z_{j}>0\right\} \Longrightarrow z_{i}=x_{i} \quad \text { for all } z \in Z(N, t, x)
$$

We say that $\rho$ is priority-free if there is no such pair of agents.

Priority-freeness is a mild equity requirement: in particular, it is implied by Equal Treatment of Equals (2).

In our next definition, we combine probabilistic rationing methods for various sets $\mathcal{N}$ of potential agents. To emphasize this dependence, we write $\rho(\mathcal{N})$ instead of $\rho$.

We define the composition of a family of methods $\rho(\mathcal{M})$ over pairwise distinct sets $\mathcal{M}$, by a priority ordering over these sets. We are given a set $\mathcal{N}$ and a preordering $\succeq$ of $\mathcal{N}$, namely a complete and transitive binary relation on $\mathcal{N}$. We denote by $\mathcal{M}$ an indifference class of $\succeq$ and by $\succ$ the strict component of $\succeq(i \succ j$ iff $i \succeq j$ but not $j \succeq i)$ capturing the pairs $i, j$ such that $i$ has priority over $j$ (Definition 4).

Lemma 5

Given are $\mathcal{N}$, a preordering $\succeq$ of $\mathcal{N}$, and for each indifference class $\mathcal{M}$ of $\succeq$, a method $\rho(\mathcal{M}) \in \mathcal{R}(\mathcal{M})$. There is a unique method $\rho(\mathcal{N}) \in \mathcal{R}(\mathcal{N})$ such that

- for all $i, j \in \mathcal{N}$, if $i \succ j$ then $\rho(\mathcal{N})$ gives priority to $i$ over $j$,

- for each equivalence class $\mathcal{M}$ of $\succeq, \rho(N)$ projects onto $\rho(\mathcal{M})$ :

for all $N \subseteq \mathcal{M}, x \in \ddot{I}^{N}$ and $t: \rho(\mathcal{M})(N, t, x)=\rho(\mathcal{N})(N, t, x)$

We call this method the $\succeq-$ priority composition of the methods $\rho(\mathcal{M})$.

The above definition is introduced by Moulin [2000] for deterministic, realvalued rationing methods.

The proof of Lemma 5 is constructive. Starting with an arbitrary problem $(N, t, x)$, observe that the restriction of $\succeq$ on the finite set $\mathrm{N}$ amounts to an 
ordered partition $N_{1}, \ldots, N_{K}$, where $N_{k}$ are the indifference classes and $N_{k} \succ$ $N_{k+1}$ for $k=1, \ldots K-1$. Denote by $\mathcal{M}_{k}$ the indifference class of $\succeq$ in $\mathcal{N}$ containing $N_{k}$ and define the non-decreasing sequence $t^{k}=\sum_{l=1}^{k} x_{N_{l}}$. Then $y=\rho(\mathcal{N})(N, t, x)$ is defined as follows when $t$ is such that $t^{k-1} \leq t \leq t^{k}$ :

$\circ y_{i}=x_{i}$ with probability 1 if $i \in \cup_{l=1}^{k-1} N_{l}$

$\circ y_{i}=0$ with probability 1 if $i \in \cup_{l=k+1}^{K} N_{l}$

$\circ y_{\left[N_{k}\right]}=\rho\left(\mathcal{M}_{k}\right)\left(N_{k}, t-t^{k-1}, x_{\left[N_{k}\right]}\right) \quad\left(\right.$ where $z_{[s]}$ is the projection of $z$ on $\left.\ddot{I}^{S}\right)$

Lemma 6

Notations as in Lemma 5. If for each indifference class $\mathcal{M}$ of $\succeq$, the method $\rho(\mathcal{M}) \in \mathcal{R}(\mathcal{M})$ meets Consistency (resp. Lower Composition, resp. Upper Composition), so does the $\succeq-$ priority composition of these methods.

We omit the straightforward, if notationally tedious, proof. Now to our main result.

Theorem 2

i) Given $\mathcal{N}$, let $\succeq$ be a preordering of $\mathcal{N}$ and for every indifference class $\mathcal{M}$ of $\succeq$, let $\rho(\mathcal{M})$ be a method in $\mathcal{R}(\mathcal{M})$ such that:

$\circ$ if $|\mathcal{M}| \geq 3 \quad \rho(\mathcal{M})$ is the proportional method

- if $|\mathcal{M}|=2 \quad \rho(\mathcal{M})$ is a method $\rho^{\lambda}$ (see Lemma 2) for some $\lambda, 0<\lambda<1$

Then the $\succeq-$ priority composition of the methods $\rho(\mathcal{M})$ meets Consistency, Upper and Lower Composition.

ii) Conversely, any method $\rho(\mathcal{N})$ in $\mathcal{R}(\mathcal{N})$ meeting these three properties obtains in this way.

The proof is in the Appendix.

Thus a method meeting CSY, LC and UC is essentially determined by its priority preordering $\succeq$ : if the latter has no equivalence class with exactly two agents, then the method is indeed determined; on each indifference class of size 2 , we have one degree of freedom, namely the choice of $\lambda$ in $] 0,1[$.

Upon adding the equity requirement of Priority-freeness, Theorem 1 delivers a characterization of the proportional method.

Corollary to Theorem 2

Assume $|\mathcal{N}| \geq 3$. The proportional method is characterized by the combination of four axioms: Consistency, Lower and Upper Composition and Priorityfreeness.

\section{Positive shares/risks, and two more characterization results}

We introduce two dual requirements for probabilistic rationing methods, that bound respectively from below and from above the expected share of an agent. Positive Share says that, unless there is nothing to distribute, any agent with a positive claim should receive something with some positive probability; Positive Risk says that an agent with a positive claim should not receive his full claim with probability one, as long as there is a deficit to cover: 
Positive Share:

$$
\left\{x_{i}>0 ; t>0\right\} \Longrightarrow E \rho_{i}(N, t, x)>0 \text { for all } N, t, x \text { and } i
$$

Positive Risk:

$$
\left\{x_{i}>0 ; t<x_{N}\right\} \Longrightarrow E \rho_{i}(N, t, x)<x_{i} \quad \text { for all } N, t, x \text { and } i
$$

Checking that the two axioms are dual of each other is easy and omitted.

Positive Share is an individual rationality condition: a positive claim, however small, must command some resources, the agent holding the claim cannot be entirely barred from the benefit of the "pie"; dually, Positive Risk says that an agent with a positive claim must contribute some resources to cover the common deficit. Both axioms are mild equity properties: a priority method $r^{\sigma}$ does not meet either axiom, precisely because it ignores entirely the claim of an agent as long as that of an agent with higher priority is not satisfied in full.

The random priority method meets both Positive Share and Positive Risk; so does the proportional method. The latter method, unlike the former one, meets the stronger property that we call Full Support : the support of the random variable $\rho(N, t, x)$ is the entire set $Z(N, t, x)$. Everything is possible with some positive probability.

Theorem 3

Assume $|\mathcal{N}| \geq 3$. The proportional method is characterized in $\mathcal{R}(\mathcal{N})$ by the two following (dual) combinations of axioms:

i) Consistency, Lower Composition and Positive Share;

ii) Consistency, Upper Composition and Positive Risk.

Note that Positive Share and Positive Risk are both logically independent of Equal Treatment of Equals (as the examples discussed in the next section make clear).

It remains to probe the tightness of our four characterizations results: Theorem 1, Theorem 2 and its Corollary, Theorem 3. To do so we introduce several egalitarian rationing methods that are interesting in their own right.

\section{Four egalitarian probabilistic rationing methods}

We set first a piece of notation. For any $N$ in $\mathcal{N}$ and any $z$ in $\ddot{I}^{N}$, we write

$$
M(N, z)=\left\{i \in N \mid z_{i}>0\right\} ; P(N, z)=\left\{i \in N \mid z_{i} \geq z_{j} \text { for all } j \in N\right\}
$$

An important consequence of Lemma 1 is that for any given incremental gain distribution there is a unique method in $\mathcal{R}$ meeting Lower Composition; dually, there is a unique method with a given incremental loss distribution and meeting Upper Composition. 
Formally, let $\alpha$ (resp. $\beta$ ) be an arbitrary mapping associating to any $(N, x)$ a probability distribution $\alpha(N, x)$ (resp. $\beta(N, x))$ on $M(N, x)$. This is a mild abuse of notation: recall that $\alpha(N, x)$ and $\beta(N, x)$ are defined as probability distributions on $Z(N, 1, x)$, namely the set of vectors $e^{i}, i \in M(N, x)$. Then the two equivalent definitions (12) (13) give the unique method $\rho$ with incremental gain $\alpha$ and meeting LC (resp. (14) (15) give the unique $\rho$ with incremental loss $\beta$ and meeting UC).

With these facts in mind, we introduce our four new methods.

Definition 6

- The equal chance of gains (in short, ecg) method meets LC and its incremental gain $\alpha(N, x)$ is the uniform distribution over $M(N, x)$, for all $N, x$.

- The equal chance of losses (ecl) method meets $U C$ and its incremental loss $\beta(N, x)$ is the uniform distribution over $M(N, x)$, for all $N, x$.

- The random uniform gains (rug) method meets $U C$ and its incremental loss $\beta(N, x)$ is the uniform distribution over $P(N, x)$.

- The random uniform losses ( rul) method meets $L C$ and its incremental gain $\alpha(N, x)$ is the uniform distribution over $P(N, x)$.

Recall that the rug method is introduced by Sasaki [1996] who speaks of the Random uniform allocation mechanism. Moulin and Stong [2000a] call this method Fair Queuing by reference to a terminology originating in the network literature.

All four methods in Definition 6 are fair in the sense of Equal Treatment of Equals (2). Two of them -ecg and rug-apply the general idea of equalizing the shares $z_{i}$ irrespective of the differences in claim sizes, and the other two $-e c l$ and $r u l$ - equalize the losses $x_{i}-z_{i}$ instead.

From Lemma 1 (statement i), ecg and ecl are dual of each other, and so are rug and rul. Before reviewing the four methods in the light of our axiomatic properties, we give an example for $N=\{1,2\}$ and $\bar{x}=(5,3)$ showing how they differ from one another.

The ecg method distributes probabilities across $Z(t, \bar{x})$ as a binomial distribution for $t=1,2,3$; for instance, $t=3$ yields

$$
e c g(2, \bar{x})=\frac{1}{4}(2,0)+\frac{1}{2}(1,1)+\frac{1}{4}(0,2)
$$

For higher $t$, ecg follows a binomial distribution on

$\left\{z \mid z_{1}+z_{2}=t\right.$ and $\left.z_{i} \geq 0\right\}$ truncated in the interval $Z(t, \bar{x})$; for instance $t=6$ gives

$$
\operatorname{ecg}(6, \bar{x})=\frac{7}{64}(5,1)+\frac{15}{64}(4,2)+\frac{42}{64}(3,3)
$$

The probability distribution of $e c l=e c g^{*}$ obtains by symmetry around $\bar{x} / 2$. Thus they are binomial for $t=6,7,8$, and truncated binomial for smaller $t s$ : 


$$
\begin{aligned}
& \operatorname{ecl}(2, \bar{x})=\frac{42}{64}(2,0)+\frac{15}{64}(1,1)+\frac{7}{64}(0,2) \\
& \operatorname{ecl}(6, \bar{x})=\frac{1}{4}(5,1)+\frac{1}{2}(4,2)+\frac{1}{4}(3,3)
\end{aligned}
$$

The $e c g$ and $e c l$ methods have the full support property: every point in $Z(t, \bar{x})$ has some chance of being reached. By contrast, rug and rul never charge more than 2 points in our example, and this holds true for any rationing problem with 2 agents (see the general formula below). To see this for rug, consider the decreasing random path (17) when $\beta(z)$ is the uniform distribution over $P(z)$. Starting from $\bar{x}$ where $P(\bar{x})=\{1\}$ the path deterministically takes away the 2 first units from agent 1 ; upon reaching $(3,3)$ we have $P(3,3)=\{1,2\}$ therefore both $(2,3)$ and $(3,2)$ are equiprobable; but the next unit of deficit brings us back to $(2,2)$ with probability one: at $(2,3), P=\{2\}$ so agent 2 loses the next unit for sure whereas at $(3,2)$ agent 1 is the sure loser. Thus the probability distribution $\operatorname{rug}(t, \bar{x})$ is actually deterministic for all but three values of $t$ :

$$
\begin{aligned}
\operatorname{rug}(t, \bar{x}) & =\left(\frac{t}{2}, \frac{t}{2}\right)(w \cdot \text { proba. } 1) \text { for } t=0,2,4,6 \\
& =\frac{1}{2}\left(\frac{t+1}{2}, \frac{t-1}{2}\right)+\frac{1}{2}\left(\frac{t-1}{2}, \frac{t+1}{2}\right) \text { for } t=1,3,5 \\
& =(t-3,3)(w \cdot p r o b a .1) \text { for } t=6,7,8
\end{aligned}
$$

The dual formulas for $r u l(t, \bar{x})$ are omitted for brevity.

Back to the general case, we note that a general formula for the probability distributions ecg $(N, t, x)$ and ecl $(N, t, x)$ is combinatorially involved, except for the "interior" allocations in $Z(N, t, x)$ :

$$
\text { if } z_{i}<x_{i} \text { for all } i: \operatorname{ecg}(N, t, x)(z)=\frac{1}{n^{t}} \frac{t !}{\prod_{i} z_{i} !}
$$

Besides the properties defining these two methods (i.e., ecg meets LC and ecl meets UC), they both meet Positive Share and Positive Risk (by Full Support). But that is all: the ecg method fails UC and CSY; the ecl method fails LC and CSY.

To check that ecg fails UC, take for instance $N=\{1,2\}, x=(2,1), t=1$, $t^{\prime}=2$; to check that it fails CSY, take $N=\{1,2,3\}, x=(2,1,1), t=2, i=3$, and any $z$ in $Z(N, t, x)$.

The situation is more interesting for the pair rug, rul.

Lemma 7

i) The expected shares of rug and rul are the following familiar (deterministic, real-valued) methods: 


$$
\begin{aligned}
& E \operatorname{ug}_{i}(N, t, x)=\min \left\{\lambda, x_{i}\right\} \quad \text { where } \lambda \text { solves } \sum_{N} \min \left\{\lambda, x_{i}\right\}=t \\
& E u_{i}(N, t, x)=\left(x_{i}-\lambda\right)_{+} \quad \text { where } \lambda \text { solves } \sum_{N} \min \left(x_{i}-\lambda\right)_{+}=t
\end{aligned}
$$

with the notation $(a)_{+}=\max (a, 0)$

ii) the rug and rul methods are Consistent;

iii) the rug method fails $L C$ and the rul method fails $U C$;

iv) the rug method meets Positive Share but fails Positive Risk; the rul method meets Positive Risk but fails Positive Share.

Proof sketch

Statement $i$

Fix $x$, and assume $x_{1} \leq x_{2} \leq \ldots \leq x_{n}$. The critical values for $t$ in system (23) are those where the set of agents for which $\lambda<x_{i}$ increases, namely $t^{1}=n x_{1}$, $t^{2}=x_{1}+(n-1) x_{2}, \ldots, t^{n}=x_{1}+\ldots+x_{n}$. The desired equation (23) obtains by inspecting the decreasing random path (14) of rug, successively on the (integer) intervals $\left[t^{n-1}, t^{n}\right],\left[t^{n-2}, t^{n-1}\right]$, and so on.

Statement $i i$

This is checked with the explicit formula for the probability distribution rug $(N, t, x)$ given below.

Statement iii

Denote by $\mathcal{R}_{u}$ the set of all methods in $\mathcal{R}$ of which the expected shares are given by (23). Check that any method in $\mathcal{R}_{u}$ must have the following incremental gain and loss:

$\alpha(N, x)=$ uniform distribution on $M(N, x)$

$\beta(N, x)=$ uniform distribution on $P(N, x)$

But ecg is the only method in $\mathcal{R}$ with this incremental loss and meeting LC; it is not in $\mathcal{R}_{u}$ (see the numerical example above). Therefore no method in $\mathcal{R}_{u}$ can meet LC; and rug is the only method in $\mathcal{R}_{u}$ meeting $\mathrm{UC}$.

Statement iv

The negative statement is clear in the numerical example. The positive one follows from the computation of the incremental gain of rug in the previous step of the proof.

\section{Remark 2}

The proof of statement iii shows that a method in $\mathcal{R}_{u}$ cannot satisfy LC; on the other hand, rug meets CSY and UC. Therefore it is the one method in $\mathcal{R}_{u}$ meeting the largest possible subset of our three invariance axioms. A dual statement holds for rul.

Next we give the explicit computation of the probability distribution rug $(N, t, x)$ (and by duality, of $r u l(N, t, x))$. This formula is due to Sasaki [1996]. 
For any $N, \lambda, x$ where $\lambda$ is an integer and $\lambda \leq \max _{i} x_{i}$, we use the notation:

$$
Q(N, \lambda, x)=\left\{i \in N \mid x_{i} \leq \lambda\right\} \quad \text { and } q(N, \lambda, x)=|Q(N, \lambda, x)|
$$

Check that for any rationing problem $(N, t, x)$ (where $0 \leq t \leq x_{N}$ ), there is a unique integer $\lambda^{*}$ solving the equation (where we omit the variable $N$ for simplicity):

$\lambda=\left\lfloor\frac{t-x_{Q(\lambda, x)}}{n-q(\lambda, x)}\right\rfloor$ where $\lfloor w\rfloor$ is the largest integer bounded above by $w$

Next, define a subset of $Z(N, t, x)$ as follows:

$$
U G(N, t, x)=\left\{\begin{array}{c}
z \in Z(N, t, x) \mid z_{i}=x_{i} \text { for all } i \in Q\left(N, \lambda^{*}, x\right), \quad z_{i}=\lambda^{*} \\
\text { or } \lambda^{*}+1 \text { otherwise }
\end{array}\right\}
$$

The probability distribution $u g(N, t, x)$ is simply the uniform distribution over $\mathrm{UG}(N, t, x)$.

One computes easily the cardinality of this set:

$$
\begin{aligned}
|\mathrm{UG}(N, t, x)| & =\frac{(a+b) !}{a ! b !} \text { where } a+b=n-q\left(\lambda^{*}, x\right) \\
\text { and } a & =t-x_{Q\left(\lambda^{*}, x\right)}-\lambda^{*} .\left(n-q\left(\lambda^{*}, x\right)\right)
\end{aligned}
$$

Finally, we use the four egalitarian methods to show that most of the characterization results are tight.

a) Theorem 1

The ecg and rul methods meet LC and ETE. Dually, ecl and rug meet UC and ETE.

The random priority method is self-dual (and has ETE).

b) Theorem 2 and Corollary

The rul method meets CSY, LC and Priority-freeness; the rug method meets CSY, UC and Priority-freeness. The family $\rho^{\lambda}$ shows that the combination LC, $\mathrm{UC}$ and Priority-freeness is not enough to characterize the proportional method for $|\mathcal{N}|=2$. The results in Moulin and Stong [in progress] imply the same statement for any $\mathcal{N}$.

c) Theorem 3

The ecg method satisfies LC and Positive Share; the ecl method has UC and Positive Risk. Here is a consistent method meeting Full Support (hence Positive Share and Positive Risk): $\rho(N, t, x)$ is the uniform distribution over $Z(N, t, x)$. One computes easily:

$$
\rho(N, t, x)\left(Y_{i}=z_{i}\right)=\frac{\left|Z\left(N \backslash i, t-z_{i,} x_{-i}\right)\right|}{|Z(N, t, x)|}
$$

so that Consistency follows at once. 


\section{References}

[1] Aumann, R. J and M. Maschler. 1985. "Game Theoretic Analysis of a Bankruptcy Problem from the Talmud," Journal of Economic Theory 36, 195-213.

[2] Balinski, M. and N. Shahidi. 1997. "A Simple Approach to the Product Rate Variation Problem via Axiomatics", mimeo, Ecole Polytechnique, Paris.

[3] Banker, R. 1981. "Equity Considerations in Traditional Full Cost Allocation Practices: An Axiomatic Perspective". In Joint Cost Allocations, S. Moriarty, Ed., Oklahoma City: University of Oklahoma Press, 110-130.

[4] G. Cachon and M. Larivière. 1996. "Capacity Choice and Allocation: Strategic Behavior and Supply Chain Performance", mimeo, Duke University, Fuqua School of Business.

[5] Demers, A, S. Keshav, and S. Shenker. 1990. "Analysis and Simulation of a Fair Queuing Algorithm". Internetworking: Research and Experience, 1, $3-26$.

[6] Elster, J. 1992. Local Justice, New York: Russell Sage Foundation.

[7] Herrero, C. 1998. "Minimal Rights and Claims Problems", mimeo, University of Alicante, Spain.

[8] Moulin, H. 1987. "Equal or Proportional Division of a Surplus, and Other Methods," International Journal of Game Theory, 16, 3, 161-186.

[9] Moulin, H. 1995. "On Additive Methods to Share Joint Costs," Japanese Economic Review, 46, 4, 303-332.

[10] Moulin, H. 2000. "Priority Rules and Other Asymmetric Rationing Methods," Econometrica, 68, 3 (May, 2000), 643-684.

[11] Moulin, H. 1999. "Axiomatic Cost and Surplus-Sharing", forthcoming in the Handbook of Social Choice and Welfare, Arrow, Sen and Suzumura, Editors, North-Holland.

[12] Moulin, H. and R. Stong. 2000a. "Fair Queuing and other Probabilistic Allocation Methods", mimeo, Rice University, http://www.ruf.rice.edu/ econ/papers/index.html.

[13] Moulin, H. and R. Stong. 2000b. "Filling urns fairly and rationally: the Polya-Eggenberger methods", in preparation.

[14] Van den Nouweland, A., J. Potters, S. Tijs and J. Zarzuelo, 1995. "Cores and Related Solution Concepts for Multi-Choice Games", ZOR - Mathematical Methods of Operations Research, 41, 289-311. 
[15] O'Neill, B. 1982. "A Problem of Rights Arbitration from the Talmud," Mathematical Social Sciences 2, 345-371.

[16] Sasaki, H. 1996. "Randomized Uniform Allocation Mechanism and SinglePeaked Preferences of Indivisible Good", mimeo, Waseda University, Japan.

[17] Sprumont Y. 1991. "The Division Problem with Single-Peaked Preferences: A Characterization of the Uniform Allocation Rule," Econometrica, 59, 2, 509-519.

[18] Sprumont Y. and Y. T. Wang. 1998. "A Characterization of the AumannShapley Method in the Discrete Cost-Sharing Model, mimeo, Université de Montréal.

[19] Thomson, W. 1995. "Axiomatic Analyses of Bankruptcy and Taxation Problems: A Survey," mimeo, University of Rochester.

[20] Wang, Y. T. 1999. "The Additivity and Dummy Axioms in the Discrete Cost-Sharing Model," forthcoming, Economic Letters.

[21] Young, H. P. 1988. "Distributive Justice in Taxation," Journal of Economic Theory, 48, 331-335.

[22] Young, H. P. 1990. "Progressive Taxation and Equal Sacrifice," American Economic Review, 80, 1, 253-266. 


\section{Appendix}

\section{Proof of Lemma 2}

By Lemma 1 there is a unique method $\rho$ in $\mathcal{R}$ meeting LC and with incremental gain $\alpha^{\lambda}$; there is also a unique $\rho^{\prime}$ in $\mathcal{R}$ meeting UC and with incremental loss $\beta^{\lambda}$. We show $\rho(t, x)=\rho^{\prime}(t, x)$ for all $t, x$, by induction on $x_{1}+x_{2}$. There is nothing to prove if $x_{1}$ or $x_{2}$ is zero, so we assume $x_{i}>0, i=1,2$. If $x_{1}+x_{2}=1 \Longleftrightarrow x_{1}=x_{2}=1$, formulas (18) and (19) yield

$$
\rho(1,(1,1))=\rho^{\prime}(1,(1,1))=\mu \cdot(1,0)+\lambda \cdot(0,1)
$$

Next we assume the desired equality holds up to $m$ and pick $\left(x_{1}, x_{2}\right)$ such that $x_{1}+x_{2}=m+1$ and $x_{i}>0, i=1,2$. We fix $t$ and $z \in Z(t, x)$ and apply (7) with $t^{\prime}=1$ :

$$
\rho(t, x)(z)=\alpha_{1}^{\lambda}(x) \cdot \rho\left(t-1, x-e^{1}\right)\left(z-e^{1}\right)+\alpha_{2}^{\lambda}(x) \cdot \rho\left(t-1, x-e^{2}\right)\left(z-e^{2}\right)
$$

Note that this equality holds even when $z_{i}=0$ for $i=1$ or 2 , if we make the convention that $\rho()(z)=0$ whenever $z$ has a negative component. We maintain this convention throughout the proof.

Invoking the inductive assumption, we see that the desired equality $\rho(t, x)(z)=$ $\rho^{\prime}(t, x)(z)$ is equivalent to the following:

$$
\rho^{\prime}(t, x)(z)=\alpha_{1}^{\lambda}(x) \cdot \rho^{\prime}\left(t-1, x-e^{1}\right)\left(z-e^{1}\right)+\alpha_{2}^{\lambda}(x) \cdot \rho^{\prime}\left(t-1, x-e^{2}\right)\left(z-e^{2}\right)
$$

We prove (24) by decomposing each of the three $\rho^{\prime}$ terms with the help of (15). Note that the summation giving $\rho^{\prime}(t, x)(z)$ in (15) bears over all monotonic paths in $D(x-z)$; therefore the corresponding summations for $\rho^{\prime}\left(t-1, x-e^{1}\right)(z-$ $\left.e^{1}\right)$ and $\rho^{\prime}\left(t-1, x-e^{2}\right)\left(z-e^{2}\right)$ bear on precisely the same set of paths. We show that (24) holds for each term in this sum: for a given $d$ in $D(x-z)$ we develop the corresponding term of the right hand sum in (15); we do this for $\rho^{\prime}(t, x)(z), \rho^{\prime}\left(t-1, x-e^{1}\right)\left(z-e^{1}\right)$, and $\rho^{\prime}\left(t-1, x-e^{2}\right)\left(z-e^{2}\right)$.

In order to develop these 3 terms we write $x-z=y$, and describe the given path $d$ as a sequence of integers

$$
a_{1}^{1}, a_{1}^{2}, a_{2}^{1}, a_{2}^{2}, \ldots, a_{k}^{1}, a_{k}^{2}, \ldots, a_{K}^{1}, a_{K}^{2},
$$

defined as follows:

$$
\begin{aligned}
d(k)-d(k-1) & =e^{1} \text { for } k=1, \ldots, a_{1}^{1} ;=e^{2} \text { for } k=a_{1}^{1}+1, \ldots, a_{1}^{1}+a_{1}^{2} \\
& =e^{1} \text { for } k=a_{1}^{1}+a_{1}^{2}+1, \ldots, a_{1}^{1}+a_{1}^{2}+a_{2}^{1} ; \text { and so on }
\end{aligned}
$$


In words, the path $d$ starts by $a_{1}^{1}$ moves "toward 1", next $a_{1}^{2}$ moves "toward 2 ", next $a_{2}^{1}$ moves toward 1 and so on. Note that $a_{1}^{1}$ and/or $a_{K}^{2}$ may be zero, but all other terms are positive.

Two final pieces of notation:

$$
\gamma(u)=\lambda^{\mu}-\mu^{\mu} \text { for all } u \in \ddot{I} ; \text { and } s_{i}=x_{i}-z_{i}
$$

Now we develop the term corresponding to the path $d$ in the right-hand sum (15)

$$
\delta(x, z)=\prod_{k=1}^{s_{12}} \beta^{\lambda}(z+d(k))(d(k)-d(k-1))
$$

taking into account the representation of $d$ by the sequence (25).

After rearranging the terms in the numerator and denominator, we get

$$
\delta(x, z)=\frac{\left(\prod_{k=1}^{s_{1}} \gamma\left(z_{1}+k\right)\right) \cdot\left(\prod_{k=1}^{s_{2}} \gamma\left(z_{2}+k\right)\right)}{\prod_{k=1}^{s_{12}} \gamma\left(z_{12}+k\right)} \cdot \lambda^{s_{1} z_{2}+\theta} \cdot \mu^{s_{2} z_{1}+\varphi}
$$

where the integers $\theta$ and $\varphi$ depend only upon $d$, not on $z$ or $x$. For instance

$$
\theta=a_{2}^{1} \cdot a_{1}^{2}+a_{3}^{1} \cdot\left(a_{1}^{2}+a_{2}^{2}\right)+\ldots+a_{k}^{1}\left(a_{1}^{2}+\ldots+a_{k-1}^{2}\right)+\ldots
$$

It is now a simple matter to compute the corresponding terms in the summations for $\rho^{\prime}\left(t-1, x-e^{1}\right)\left(z-e^{1}\right)$ and $\rho^{\prime}\left(t-1, x-e^{2}\right)\left(z-e^{2}\right)$.

$$
\begin{aligned}
& \delta\left(x-e^{1}, z-e^{1}\right)=\delta(x, z) \cdot \frac{\gamma\left(z_{12}+s_{12}\right)}{\gamma\left(z_{12}\right)} \cdot \frac{\gamma\left(z_{1}\right)}{\gamma\left(z_{1}+s_{1}\right)} \cdot \frac{1}{\mu^{s_{2}}} \\
& \delta\left(x-e^{2}, z-e^{2}\right)=\delta(x, z) \cdot \frac{\gamma\left(z_{12}+s_{12}\right)}{\gamma\left(z_{12}\right)} \cdot \frac{\gamma\left(z_{2}\right)}{\gamma\left(z_{2}+s_{2}\right)} \cdot \frac{1}{\lambda^{s_{1}}}
\end{aligned}
$$

The verification that

$\delta(x, z)=\alpha_{1}^{\lambda}(x) . \delta\left(x-e^{1}, z-e^{1}\right)+\alpha_{2}^{\lambda}(x) . \delta\left(x-e^{2}, z-e^{2}\right)$ is now immediate.

\section{Proof of Lemma 3}

Recall the following property, for any method $\rho$ in $\mathcal{R}$ with incremental loss/gain $\alpha, \beta$

$$
\alpha_{1}(1,1)=\beta_{2}(1,1) ; \alpha_{2}(1,1)=\beta_{1}(1,1)
$$


(the property was used in the proof of statement ii in Theorem 1; it follows at once from (10) (11)).

Lemma 2 shows that all methods in the family $\rho^{\lambda}$ satisfy LC and UC. Now we start from a method $\rho$ meeting LC and UC, and with incremental gain/loss distributions $\alpha, \beta$ : we must show that $\rho$ is in the family $\rho^{\lambda}$.

Consider the system (16) (17). With the usual convention that a negative coordinate makes the corresponding probability zero, this system writes as follows for any $x \in \ddot{I}^{(12)}$ :

$$
\begin{aligned}
& \beta_{1}(x)=\alpha_{1}(x) \cdot \beta_{1}\left(x_{1}-1, x_{2}\right)+\alpha_{2}(x) \cdot \beta_{1}\left(x_{1}, x_{2}-1\right) \\
& \alpha_{2}(x)=\beta_{1}(x) \cdot \alpha_{2}\left(x_{1}-1, x_{2}\right)+\beta_{2}(x) \cdot \alpha_{2}\left(x_{1}, x_{2}-1\right)
\end{aligned}
$$

Our next step is to show that the system (27) (28) determines entirely $\alpha$ and $\beta$ from the single number $\lambda=\alpha_{2}(1,1)=\beta_{1}(1,1)$. We have $0 \leq \lambda \leq 1$ and we deal with the cases $\lambda=0,1$ first. Assume $\lambda=0$. From (27) applied to $\left(1, x_{2}\right)$ we get

$$
\begin{aligned}
& \beta_{1}\left(1, x_{2}\right)=\alpha_{2}\left(1, x_{2}\right) \cdot \beta_{1}\left(1, x_{2}-1\right) \\
& \left.\alpha_{2}\left(1, x_{2}\right)=\beta_{1}\left(1, x_{2}\right) \cdot \beta_{2}\left(1, x_{2}\right) \cdot \alpha_{2}\left(1, x_{2}-1\right)\right]
\end{aligned}
$$

from which it follows easily (by induction on $x_{2}$ ) that $\beta_{1}\left(1, x_{2}\right)=\alpha_{2}\left(1, x_{2}\right)=$ 0 for all $x_{2}>0$. One checks similarly $\beta_{1}\left(x_{1}, 1\right)=\alpha_{2}\left(x_{1}, 1\right)=0$ for all $x_{1}>0$. Finally, by induction on $x_{1}+x_{2}$, system (27) (28) yields $\beta_{1}(x)=\alpha_{2}(x)=0$ for all $x>>0$. Thus for $\lambda=0$ the method $\rho$ is the priority method where 1 precedes 2. Similarly, for $\lambda=1$, we find $\beta_{1}(x)=\alpha_{2}(x)=1$ for all $x$ s.t. $x_{i}>0$, $i=1,2$, namely $\rho$ is the other priority method.

From now on we assume $0<\lambda<1$. We prove simultaneously that $\alpha_{2}(x)$ and $\beta_{1}(x)$ are uniquely determined and that

$$
\text { for all } x>>0 \quad 0<\alpha_{2}(x)<1 ; \quad 0<\beta_{1}(x)<1
$$

We proceed by induction, and take care of the cases $\left(1, x_{2}\right)$ and $\left(x_{1}, 1\right)$ first.

For $x=\left(1, x_{2}\right), \quad x_{2} \geq 2$, consider system (29). If $a=\alpha_{2}\left(1, x_{2}-1\right)$ and $b=\beta_{1}\left(1, x_{2}-1\right)$ are both in $] 0,1[,(29)$ delivers a unique solution

$$
\begin{aligned}
\beta_{1}\left(1, x_{2}\right)= & \frac{a b}{1-b(1-a)}, \quad \alpha_{2}\left(1, x_{2}\right)=\frac{a}{1-b(1-a)} \\
& \text { and both numbers are in }] 0,1[\text { as desired. }
\end{aligned}
$$

A similar argument takes care of the case $x=\left(x_{1}, 1\right)$. 
Next we prove the claim for all $x>>0$, by induction on $x_{1}+x_{2}$. By assumption, the claim is true for $x_{1}+x_{2}=2$. Consider next an arbitrary $x$ such that $x \geq(2,2)$ (if $x_{i}=1$ for $i=1$ or 2 , the claim is already established). We set $b_{1}=\beta_{1}\left(x_{1}-1, x_{2}\right), b_{2}=\beta_{1}\left(x_{1}, x_{2}-1\right), a_{1}=\alpha_{2}\left(x_{1}-1, x_{2}\right), a_{2}=\alpha_{2}\left(x_{1}, x_{2}-1\right):$ the inductive assumption gives $0<b_{i}, a_{i}<1$ for $i=1,2$. Next we eliminate $\alpha_{1}(x)=1-\alpha_{2}(x)$ and $\beta_{2}(x)=1-\beta_{1}(x)$ from system (27) (28) which becomes:

$$
\begin{aligned}
\beta_{1}(x)+\left(b_{1}-b_{2}\right) \cdot \alpha_{2}(x) & =b_{1} \\
\left(a_{2}-a_{1}\right) \beta_{1}(x)+\alpha_{2}(x) & =a_{2}
\end{aligned}
$$

The determinant of this system is strictly positive:

$$
\left\{-1<b_{1}-b_{2}<1,-1<a_{2}-a_{1}<1\right\} \Longrightarrow 1-\left(b_{1}-b_{2}\right)\left(a_{2}-a_{1}\right)>0
$$

therefore $\beta_{1}(x)$ and $\alpha_{2}(x)$ are uniquely determined

$$
\beta_{1}(x)=\frac{b_{1}-a_{2}\left(b_{1}-b_{2}\right)}{1-\left(b_{1}-b_{2}\right) \cdot\left(a_{2}-a_{1}\right)} ; \alpha_{2}(x)=\frac{a_{2}-b_{1}\left(a_{2}-a_{1}\right)}{1-\left(b_{1}-b_{2}\right) \cdot\left(a_{2}-a_{1}\right)}
$$

It remains to check that both numbers are in $] 0,1[$. This results at once from the fact that $a_{i}, b_{i}$ are in that interval, too, and from (30)

$$
\begin{aligned}
& \beta_{1}(x)>0 \Longleftrightarrow\left(1-a_{2}\right) \cdot b_{1}+a_{2} \cdot b_{2}>0 \\
& \alpha_{2}(x)>0 \Longleftrightarrow\left(1-b_{1}\right) \cdot a_{2}+b_{1} \cdot a_{1}>0 \\
& \beta_{1}(x)<1 \Longleftrightarrow\left(1-a_{1}\right) \cdot b_{1}+a_{1} \cdot b_{2}<1 \\
& \alpha_{2}(x)<1 \Longleftrightarrow\left(1-b_{2}\right) \cdot a_{2}+b_{2} \cdot a_{1}<1
\end{aligned}
$$

Summing up our findings: for any number $\lambda, 0 \leq \lambda \leq 1$, we have shown that there is at most one method $\rho$ in $\mathcal{R}$, meeting LC, UC and such that $\alpha_{2}(1,1)=\beta_{1}(1,1)=\lambda$.

On the other hand, the method $\rho^{\lambda}$ defined for all $\lambda, 0 \leq \lambda \leq 1$, (see Lemma 3 and the discussion thereafter) meets LC and UC. It is immediate to check from (18) (19) that $\alpha^{\lambda}(1,1)(2)=\beta^{\lambda}(1,1)(1)=\lambda$, and the cases $\lambda=0, \frac{1}{2}, 1$ are equally clear.

The conscientious reader may also check that the formulas (18) (19) for $\alpha_{2}^{\lambda}(x)$ and $\beta_{1}^{\lambda}(x)$ deliver indeed the unique solution of system (27) (28) starting at $\lambda$.

Remark 3

We noted in Section 4 that a convex combination of priority methods fails both LC and UC: see the discussion after Lemma 1. This is a general feature of the $\mathrm{LC}$ and UC axioms. 
Let $\rho^{1}, \rho^{2}$ be two methods in $\mathcal{R}$ meeting LC, and $\rho=\lambda \rho^{1}+\mu \rho^{2}$ be a proper convex combination, for some $\lambda, 0<\lambda<1$, with the usual notation $\mu=1-\lambda$. The method $\rho \in \mathcal{R}$ can be interpreted as a lottery over $\left\{\rho^{1}, \rho^{2}\right\}$ with probabilities $\lambda$ and $\mu$ respectively.

We assume that $\rho$ meets LC as well and consider, for any $i$ and any $x$ s.t. $x_{i} \geq 2$, the equality

$$
\rho(2, x)\left(2 e^{i}\right)=\lambda \rho^{1}(2, x)\left(2 e^{i}\right)+\mu \cdot \rho^{2}(2, x)\left(2 e^{i}\right)
$$

By LC, each term can be computed from the incremental gain distribution, denoted respectively $\alpha, \alpha^{1}, \alpha^{2}$ :

$$
\alpha_{i}(x) \cdot \alpha_{i}\left(x-e^{i}\right)=\lambda \cdot \alpha_{i}^{1}(x) \cdot \alpha_{i}^{1}\left(x-e^{i}\right)+\mu \cdot \alpha_{i}^{2}(x) \cdot \alpha_{i}^{2}\left(x-e^{i}\right)
$$

Using $\alpha_{i}=\lambda . \alpha_{i}^{1}+\mu . \alpha_{i}^{2}$ and rearranging, we get

$$
\left(\alpha_{i}^{1}(x)-\alpha_{i}^{2}(x)\right) \cdot\left(\alpha_{i}^{1}\left(x-e^{i}\right)-\alpha_{i}^{2}\left(x-e^{i}\right)\right)=0
$$

Let $A_{i}=\left\{x \in \ddot{I}^{N} \mid \alpha_{i}^{1}(x)=\alpha_{i}^{2}(x)\right\}$. We have just shown that for all $x$, $x_{i} \geq 1$,one of $x$ or $x-e^{i}$ is in $A_{i}$ (if $x_{i}=1$ this is obvious). A similar argument for $\rho(3, x)\left(3 e^{i}\right)$ shows that for all $x, x_{i} \geq 2$, at most one of $x, x-e^{i}, x-2 e^{i}$ is not in $A^{i}$. An induction argument then gives that in any "line" $B\left(x_{-i}\right)=$ $\left\{z \in \ddot{I}^{N} \mid z_{-i}=x_{-i}\right\}$, there is at most one point outside $A^{i}$. In other words, the incremental distributions of $\rho^{1}$ and $\rho^{2}$ cannot be too different.

For simplicity, consider the two agents case, $N=\{1,2\}$. Here $A^{1}=A^{2}$, hence $\rho^{1}$ and $\rho^{2}$ can differ at most on the graph a bijection from $\ddot{I}$ into itself. Upon examining the impact of $\rho=\lambda \rho^{1}+\mu \rho^{2}$ in formula (13), we find that the set on which $\alpha^{1}$ and $\alpha^{2}$ differ must intersect any increasing path in $\ddot{I}^{2}$ at most once. Therefore it is the graph of a decreasing map from $\ddot{I} \cup\{0\}$ into itself. For instance we can have:

$$
\begin{aligned}
& \alpha^{1}(x) \neq \alpha^{2}(x) \text { for all } x \text { s.t. } x_{1}+x_{2}=10 \\
& \alpha^{1}(x)=\alpha^{2}(x) \text { elsewhere }
\end{aligned}
$$

\section{Proof of Theorem 2 and Corollary}

Statement i follows at once from Lemmas 2, 4 and 6 . The proof of statement ii proceeds in four steps.

Step 1 An auxiliary result

Assume $|\mathcal{N}| \geq 3$ and let $\rho \in \mathcal{R}(\mathcal{N})$ be a method satisfying Consistency and with an incremental gain $\alpha$ such that

for all $i, j \in \mathcal{N}$, all $x \in \ddot{I}^{\{i, j\}}: x_{i}>0 \Longrightarrow \alpha_{i}(\{i, j\}, x)>0$ 
Then there exists for all $i$ a realvalued function $f_{i}$ defined on $\ddot{I}$, with $f_{i}(0)=0, f_{i}\left(x_{i}\right)>0$ for $x_{i}>0$, and such that:

$\alpha_{i}(N, x)=\frac{f_{i}\left(x_{i}\right)}{\sum_{N} f_{j}\left(x_{j}\right)} \quad$ for all $N \subseteq \mathcal{N}, \quad$ all $i \in N \quad$ and all $x \neq 0$

Proof: for any finite subset $N$ of $\mathcal{N}$, Consistency (21) applied to $t=1$, any $x, z=e^{j}$ and any $i \neq j$ yields

$$
\alpha_{j}(N, x)=\alpha_{j}\left(N \backslash i, x_{-i}\right) \cdot\left(1-\alpha_{i}(N, x)\right)
$$

Now take $N_{0}=\{1,2,3\}$, fix $x$ such that $x_{i}>0$ for $i=1,2,3$, and write for simplicity $\alpha_{i}=\alpha_{i}\left(N_{0}, x\right)$ and $\alpha_{i}(i, j)=\alpha_{i}\left(\{i, j\},\left(x_{i}, x_{j}\right)\right)$.

Apply (32) to all six ordered pairs $(i, j)$ in $N_{0}$ :

$$
\begin{aligned}
& \alpha_{1}=\left(1-\alpha_{2}\right) \cdot \alpha_{1}(13) ; \quad \alpha_{2}=\left(1-\alpha_{3}\right) \cdot \alpha_{2}(12) ; \quad \alpha_{3}=\left(1-\alpha_{1}\right) \cdot \alpha_{3}(23) \\
& \alpha_{1}=\left(1-\alpha_{3}\right) \cdot \alpha_{1}(12) ; \quad \alpha_{2}=\left(1-\alpha_{1}\right) \cdot \alpha_{2}(23) ; \quad \alpha_{3}=\left(1-\alpha_{2}\right) \cdot \alpha_{3}(13)
\end{aligned}
$$

The assumption $\alpha_{i}(i, j)>0$ implies $\alpha_{i}<1$ for all $i$ (e.g., $\alpha_{1}=1$ brings a contradiction from $\left.\alpha_{2}=\left(1-\alpha_{3}\right) \cdot \alpha_{2}(12)\right)$. Thus we can multiply the three top equations, the three bottom ones, and simplify:

$$
\alpha_{1}(13) \cdot \alpha_{2}(12) \cdot \alpha_{3}(23)=\alpha_{1}(12) \cdot \alpha_{2}(23) \cdot \alpha_{3}(13)
$$

Equivalently

$$
\frac{\alpha_{1}}{\alpha_{2}}\left(12,\left(x_{1}, x_{2}\right)\right) \cdot \frac{\alpha_{2}}{\alpha_{3}}\left(23,\left(x_{2}, x_{3}\right)\right) \cdot \frac{\alpha_{3}}{\alpha_{1}}\left(13,\left(x_{1}, x_{3}\right)\right)=1
$$

Letting $x_{1}, x_{2}$ vary and keeping $x_{3}$ fixed, the above equation implies that $\alpha_{1} / \alpha_{2}$ takes the form

$$
\frac{\alpha_{1}}{\alpha_{2}}\left(12,\left(x_{1}, x_{2}\right)\right)=\frac{f\left(x_{1}\right)}{g\left(x_{2}\right)} \quad \text { for all } x_{1}, x_{2}=1,2 \ldots
$$

where the functions $f, g$ are realvalued and positive for all $x_{i}>0$. We can apply the same argument to $\alpha_{2} / \alpha_{3}(23)$ and $\alpha_{3} / \alpha_{1}(13)$; moreover a straightforward normalization yields three realvalued functions $f_{i}$, such that for all $i, j$ in $\{1,2,3\}$, and all $x_{i}, x_{j}>0$.

$$
\frac{\alpha_{i}}{\alpha_{j}}\left(i, j,\left(x_{i}, x_{j}\right)\right)=\frac{f_{i}\left(x_{i}\right)}{f_{j}\left(x_{j}\right)} \Longleftrightarrow \alpha_{i}\left(i j,\left(x_{i}, x_{j}\right)\right)=\frac{f_{i}\left(x_{i}\right)}{f_{i}\left(x_{i}\right)+f_{j}\left(x_{j}\right)}
$$


If $x_{i}=0$,we know that $\alpha_{i}\left(i j,\left(x_{i}, x_{j}\right)\right)=0$ therefore by extending $f_{i}$ to $f_{i}(0)=0$, we have constructed three functions $f_{1}, f_{2}, f_{3}$ as in the statement of Lemma 6 and such that (31) holds for all three subsets $\{i j\}$ of $N_{0}=\{1,2,3\}$.

It is now a simple matter to extend the definition of $f_{i}$ to all $i \in \mathcal{N}$. For any $i \in \mathcal{N} \backslash N_{0}$,apply the argument above to $\{1,2, i\}$, keeping the same functions $f_{1}, f_{2}$ just defined and defining a new $f_{i}$. For any pair $i, j$ disjoint from $\{1,2\}$, we apply the previous argument to the set $\{1, i, j\}$ to get property (31) for the pair $\{i, j\}$.

It remains to extend the property (31) from the sets $N$ of size two to all finite subsets of $\mathcal{N}$. This follows from (32) an easy induction argument on $|N|$. Assume (31) holds up to $|N|-1$, then pick $x>>0$ and an arbitrary pair $i, j$ : equation (32) writes as follows

$$
\frac{\alpha_{j}(N, x)}{f_{j}\left(x_{j}\right)}=\frac{\sum_{N \backslash i} \alpha_{k}(N, x)}{\sum_{N \backslash i} f_{k}\left(x_{k}\right)}=\frac{1}{\sum_{N} f_{k}\left(x_{k}\right)}
$$

as desired

Step 2. Another auxiliary result

Let $\rho \in \mathcal{R}(\mathcal{N})$ be a method satisfying Consistency. We write $\sup (\rho, N, x)$ for the support of the probability distribution $(\rho, N, x)$ when $t$ varies from 0 to $x_{N}$ :

$$
\sup (\rho, N, x)=\left\{z \in[0, x] \mid \rho\left(N, z_{N}, x\right)>0\right\}
$$

For all finite subsets $M, N$ of $\mathcal{N}$ with $M \subset N$ and all $x$ in $\ddot{I}^{N}$, we have:

$$
\sup \left(\rho, M, \operatorname{proj}_{M}(x)\right) \supseteq \operatorname{proj}_{M}[\sup (\rho, N, x)]
$$

where $\operatorname{proj}_{M}$ is the projection operator from $\ddot{I}^{N}$ onto $\ddot{I}^{M}$.

Pick an arbitrary element $z \in \sup (\rho, N, x)$ and an agent $\mathrm{i} \in N \backslash M$. In the Consistency equation (21), the left-hand term is positive, hence the right-hand terms are positive as well, implying $z_{-i} \in \sup \left(\rho, N \backslash i, x_{-_{i}}\right)$. Repeat the argument by successively deleting all agents in $N \backslash M$, to obtain $\operatorname{proj}_{M}(z) \in$ $\sup \left(\rho, M, \operatorname{proj}_{M}(x)\right)$, and the inclusion (33) is established.

Note that the converse inclusion holds true as well, for any method meeting Consistency and Lower and/or Upper Composition. We omit the proof.

Step 3. Constructing the priority preordering

We start now the proof of statement ii: we fix a method $\rho \in \mathcal{R}(\mathcal{N})$ meeting CSY, LC and UC. In this step we analyze the priority relation (Definition 4) of $\rho$ : we show that this relation is a preordering, and apply then Lemma 5 to decompose $\rho$ into a family of priority-free methods.

For any pair $i, j$ consider the property: " $\rho$ gives priority to $i$ over $j$," Definition 4 . By step 2, we have for all $N, x$ : 


$$
\sup \left(\rho,\{i, j\},\left\{x_{i}, x_{j}\right\}\right) \supseteq \operatorname{proj}_{\{i, j\}}[\sup (\rho, N, x)]
$$

Therefore if property (22) holds for $N=\{i, j\}$, it holds for any N. For $N=\{i, j\}$, property (22) says that the method $\rho(i j) \in \mathcal{R}(i j)$ is the priority method with $i$ first. On the other hand, $\rho(i j)$ meets LC and UC, so by Lemma $1, \rho(i j)$ is the priority with $i$ first if and only if $\alpha_{j}(i j,(1,1))=0$ (recall that the method $\rho^{\lambda}$ is entirely determined by $\left.\lambda=\alpha_{i}(1,1)\right)$.

We define the priority relation $\succsim$ of $\rho$, its strict component and indifference relation as follows:

$$
\begin{aligned}
i & \succ j \Longleftrightarrow \rho \text { gives priority to } i \text { over } j \Longleftrightarrow \alpha_{j}(i j,(1,1))=0 \\
i & \backsim j \Longleftrightarrow \rho(i j) \text { is priority-free } \quad \Longleftrightarrow 0<\alpha_{i}(i j,(1,1))<1 \\
& \Longleftrightarrow 0<\alpha_{j}(i j,(1,1))<1 \\
i & \succsim j \Longleftrightarrow \alpha_{j}(i j,(1,1))>0
\end{aligned}
$$

We show next that $\succsim$ is a preordering. It is obviously complete; for transitivity, we pick three arbitrary agents $1,2,3$ and prove the following:

$$
\left\{\alpha_{1}(12,(1,1))>0 \text { and } \alpha_{2}(23,(1,1))>0\right\} \Longrightarrow\left\{\alpha_{1}(13,(1,1))>0\right\}
$$

We use repeatedly property (32) for $N=1,2,3$, using the shorthand:

$$
\alpha_{i}=\alpha_{i}(N,(1,1,1)) \text { and } \alpha_{i}(i j)=\alpha_{i}(i j,(1,1)) .
$$

We prove (35) by contradiction. Assume the inequalities in the premises and $\alpha_{1}(13)=0$. Apply (32) three times in sequence:

$$
\begin{aligned}
& \alpha_{1}(13)=0 \text { and } \alpha_{1}=\left(1-\alpha_{2}\right) \cdot \alpha_{1}(13) \Longrightarrow \alpha_{1}=0 \\
& \alpha_{1}(12)>0 \text { and } \alpha_{1}=\left(1-\alpha_{3}\right) \cdot \alpha_{1}(12) \Longrightarrow \alpha_{3}=1 \Longrightarrow \alpha_{2}=0 \\
& \alpha_{2}(23)>0 \text { and } \alpha_{2}=\left(1-\alpha_{1}\right) \cdot \alpha_{2}(23) \Longrightarrow \text { contradiction }
\end{aligned}
$$

If the priority relation of a method $\rho$ defines a preordering $\succsim$ (the strict component of which is the relation in Definition 4), the method itself is the $\succsim$ priority composition of its projections $\rho(\mathcal{M})$ over all indifference classes of $\succsim$. This fact is just a rewording of Lemma 5 .

In the case of our method $\rho$ meeting CSY, LC and UC, we note that the methods $\rho(\mathcal{M})$ meet these three axioms as well. The additional property of $\rho(\mathcal{M})$ is to be priority-free. In the next and last step of the proof, we show that this forces the proportional method whenever $|\mathcal{M}| \geq 3$.

Step 4. The case of priority-free methods. 
All that remains to be shown is this: let $\rho \in \mathcal{R}(\mathcal{N})$ be a priority-free method meeting CSY, LC and UC; if $|\mathcal{N}| \geq 3, \rho$ must be the proportional method.

For every pair of agents $i, j$, the method $\rho(i j)$ (the projection of $\rho$ on $\{i, j\}$, see Lemma 5) meets LC and UC, therefore it is a method $\rho^{\lambda}$ from Lemma 2. Priority-freeness implies $0<\lambda<1$.

On the other hand, we can apply the auxiliary result of Step 1, and $\alpha_{i}(i j, x)$ takes the form (31) for some real-valued function $f_{i}$. We now combine these two representations of $\alpha_{i}\left(i j,\left(x_{i}, x_{j}\right)\right)$.

By definition of the methods $\rho^{\lambda}$, the ratio $\alpha_{i}(i j, x) / \alpha_{j}(i j, x)$ can only take one of two forms:

$$
\begin{aligned}
& \frac{\alpha_{i}}{\alpha_{j}}(i j, x)=\frac{x_{i}}{x_{j}} \text { if } \rho(i j) \text { is the proportional method } \\
& \frac{\alpha_{i}}{\alpha_{j}}(i j, x)=\frac{\lambda^{x_{i}}-\mu^{x_{i}}}{\lambda^{x_{i}}} \cdot \frac{\mu^{x_{j}}}{\lambda^{x_{j}}-\mu^{x_{j}}} \quad \text { otherwise. }
\end{aligned}
$$

Define $\lambda(i, j)=\frac{1}{2}$ in the former case and $\lambda(i, j)=\lambda$ in the latter. Comparing these formulas with (31) gives

$$
\begin{aligned}
& f_{i}\left(x_{i}\right)=a_{i} \cdot x_{i} \text { for some constant } a_{i}>0 \text {, if } \rho(i j) \text { is proportional } \\
& f_{i}\left(x_{i}\right)=a_{i} \cdot \frac{\lambda^{x_{i}}-\mu^{x_{i}}}{\lambda^{x_{i}}} \text { for some constant } a_{i}>0, \text { otherwise. }
\end{aligned}
$$

Define $\lambda(i, j)=\frac{1}{2}$ if $\rho(i, j)$ is the proportional method and $\lambda(i, j)=\lambda$ otherwise. The above system shows that $\lambda(i, j)$ is independent of $j$ (because $f_{i}$ is $): \lambda(i, j)=\lambda(i, k)$ for all triple $i, j, k$. It also implies $\lambda(i, j)+\lambda(j, i)=1$. These two properties, together with $|\mathcal{N}| \geq 3$, yield $\lambda(i, j)=\frac{1}{2}$ for all $i, j$. Thus $\rho(i j)$ is the proportional method for all $i, j$.

A final induction argument based on property (32) (and similar to the argument at the end of Step 1) shows that $\alpha_{i}(N, x)$ is proportional to $x_{i}$ for all $i, N, x$, and the proof of Theorem 2 is complete.

Step 5. Proof of the Corollary.

It is precisely the object of Step 4 above.

\section{Proof of Theorem 3}

Let $\rho \in \mathcal{R}(\mathcal{N})$ meet CSY, LC and Positive Share. By the result in Step 1 of the previous proof, the incremental gain distribution of $\rho$ takes the form (31) for some realvalued functions $f_{i}$ as described in the result.

We apply Consistency (21) to an arbitrary triple $N=\{1,2,3\}$ in $\mathcal{N}$ and to $i=2, t=2, x$ s.t. $x_{j} \geq 1$ for $j=1,2,3$, and $z=(1,1,0)$ :

$$
\rho(N, 2, x)(1,1,0)=\rho\left(13,1,\left(x_{1}, x_{3}\right)\right)(1,0) . \rho(N, 2, x)\left(Y_{2}=1\right)
$$

We compute the left-hand term in (36) with the help of Lower Composition (7) 


$$
\rho(N, 2, x)(1,1,0)=\alpha_{1}(N, x) \cdot \alpha_{2}\left(N, x-e^{1}\right)+\alpha_{2}(N, x) \cdot \alpha_{1}\left(N, x-e^{2}\right)
$$

Denote for simplicity $f_{i}=f_{i}\left(x_{i}\right), s=\sum_{N} f_{i}, s_{i}=f_{i}\left(x_{i}-1\right)+\sum_{N \backslash i} f_{j}$, all positive, and apply (31):

$$
\rho(N, 2, x)(1,1,0)=f_{1} \cdot f_{2} \cdot \frac{1}{s}\left(\frac{1}{s_{1}}+\frac{1}{s_{2}}\right)
$$

Similarly,

$$
\begin{aligned}
\rho(N, 2, x)\left(Y_{2}=\right. & 1)=\rho(N, 2, x)(1,1,0)+\rho(N, 2, x)(0,1,1)= \\
& f_{1} \cdot f_{2} \cdot \frac{1}{s} \cdot\left(\frac{1}{s_{1}}+\frac{1}{s_{2}}\right)+f_{2} \cdot f_{3} \cdot \frac{1}{s} \cdot\left(\frac{1}{s_{2}}+\frac{1}{s_{3}}\right)
\end{aligned}
$$

Now (36) develops as

$$
\begin{aligned}
\frac{f_{1} f_{2}}{s}\left(\frac{1}{s_{1}}+\frac{1}{s_{2}}\right) & =\frac{f_{1}}{f_{1}+f_{3}} \cdot\left[\frac{f_{1} f_{2}}{s} \cdot\left(\frac{1}{s_{1}}+\frac{1}{s_{2}}\right)+\frac{f_{2} f_{3}}{s} \cdot\left(\frac{1}{s_{2}}+\frac{1}{s_{3}}\right)\right] \\
& \Longleftrightarrow s_{1}=s_{3} \Longleftrightarrow f_{1}\left(x_{1}\right)-f_{1}\left(x_{1}-1\right)=f_{3}\left(x_{3}\right)-f_{3}\left(x_{3}-1\right)
\end{aligned}
$$

In this computation, the agents 1,3 were arbitrary, and so were $x_{1} \geq 1$ and $x_{3} \geq 1$.

Therefore $f_{i}\left(x_{i}\right)-f_{i}\left(x_{i}-1\right)=a$ is independent both of $i$ and of $x_{i} \geq 1$. As $f_{i}(0)=0$ and $f_{i}(1)>0$ for all $i$, we deduce $a>0$ and $f_{i}\left(x_{i}\right)=a . x_{i}$ for all $i$ and all $x_{i} \geq 0$. Therefore $\alpha(N, x)$ is parallel to the vector $x$, as desired.

The proof of statement $\mathrm{i}$ is complete. That of statement ii follows at once by duality. 